\begin{tabular}{|c|c|c|c|}
\hline & 51 & $38-65$ str. & Zagreb 2016. \\
LARUS & $(2016)$ & 18 slika & \\
znanatska akademija & \multicolumn{3}{|r}{} \\
\cline { 2 - 4 } & \multicolumn{2}{|r}{} \\
\hline
\end{tabular}

UDK

598.2.063.7(497.5)

Professional paper

\title{
RARE BIRDS IN CROATIA THE FOURTH REPORT OF THE CROATIAN BIRDS RARITIES COMMITTEE
}

\author{
Rijetke ptice u Hroatskoj \\ Četorti izvještaj komisije za rijetke vrste ptica
}

\author{
Sanja Barišić ${ }^{1}$, Jelena Kralj ${ }^{1}$, Luka Jurinović ${ }^{2}$ \\ ${ }^{1}$ Institute of Ornithology, Croatian Academy of Sciences and Arts, Gundulićeva \\ 24, HR-10000 Zagreb \\ ${ }^{2}$ Croatian Veterinary Institute, Poultry Centre, Heinzelova 55, HR-10000 Zagreb
}

\begin{abstract}
In the fourth report of the Croatian Birds Rarities Committee, new accepted records of rare birds that were submitted to the committee in the period 2012-2016 are reported. Ten species, of which one is treated as escape from captivity, and two subspecies have been observed in Croatia for the first time. The list of birds of Croatia now holds 396 species in categories A, B and C, and 5 species in category C E. Further 11 species are recorded in category $E$ and one in category $D$, and these are not included in the list of birds of Croatia. Three species have been removed from the rare birds list due to the increasing number of their observations. The list of rare bird taxa now includes 72 species, of which 5 are included in category $\mathrm{C} \mathrm{E}$, and 4 subspecies. Additional eleven species are treated as escapees (category E) and one as probable escapee (category D).
\end{abstract}

Keywords: rare birds, Croatia, bird list

\section{INTRODUCTION}

The $4^{\text {th }}$ report of the Croatian Birds Rarities Committee covers observations of rare bird taxa (species and subspecies) in Croatia reported to the Committee in the

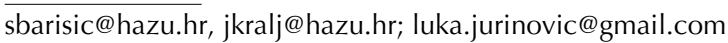


period 2012-2016. Apart from validating reported observation of bird taxa listed as rare in Croatia or bird taxa that have for the first time been observed in Croatia, the Croatian Birds Rarities Committee is also responsible for updating Croatian national bird species list. Members of the Croatian Birds Rarities Committee are: Sanja Barišić (Chair), Jelena Kralj, Krešimir Leskovar, Vladimir Bartovsky, Ivan Budinski and Luka Jurinović. For the purposes of this report, the bird taxonomy and systematics have been updated following DEL Hoyo et al. (2014).

Bird taxa listed as rare in Croatia are given systematically. English and scientific names are given after DEL Hoyo et al. (2014), while Croatian names are given after RADović et al. (2005) and the unpublished list of bird names prepared by the same authors. Numbers after the species names refer to the number of records and the number of individuals reported in the last 50 years. If the individual count is approximate, the minimum number of individuals followed by the sign + is given. The sign * next to the count numbers indicates that the only records of a bird taxa are more than 50 years old. In addition, for the observations covered by this report, detailed information is given: date (DD/MM/YYYY), locality, number of birds, age or sex if known, and the observer. Within the species, separate records are listed by date. Observations of several rare bird species that were published in Miкuska \& Miкuska (1994), Rubinič et al. (2002), Štumberger (2005), and Sušić (2015), as well as escapees published in Miкuska et al. (2002) are included in the species record counts. Rejected records are listed separately. The complete list of the birds of Croatia is given in the Appendix 1.

\section{Description of categories:}

AERC (Association of European Rarities Committees) (www.aerc.eu/guidelines.html):

A Species that have been recorded in an apparently natural state at least once since $1^{\text {st }}$ January 1950.

B Species that have been recorded in an apparently natural state but only between $1^{\text {st }}$ January 1800 and $31^{\text {st }}$ December 1949.

C Species that were originally introduced by man, but have established selfsupported breeding populations derived from introduced stock, also birds probably originating from a category $C$ population in other country.

D Species that would otherwise appear in categories A or B except that there is reasonable doubt that they have ever occurred in a natural state.

E Species that have been recorded as escapees from captivity.

\section{Occurrence}

$\mathrm{R} \quad$ Regular - species recorded in at least 7 years out of the last 10 years

Ir Irregular - species recorded more than 10 times and in more than 5 years in the last 50 years but in fewer than 7 years out of the last 10 years

Rare-species recorded $1-10$ times or in $1-5$ years in the last 50 years 
From December 2012 to October 2016, Croatian Birds Rarities Committee examined 98 records, 96 of which were accepted. Compared to previous report that covered years 2005 - 2012 (KRALJ \& BARIŠIĆ 2013), the number of rare species observations recorded per year increased from 17.5 to 22.5 , which points to a moderate increase in bird-watching activity in Croatia in recent years.

Ten species have been observed in Croatia for the first time: Barnacle Goose Branta leucopsis, Canada Goose Branta canadensis, White-cheeked Pintail Anas bahamensis, American Golden Plover Pluvialis dominica, Pectoral Sandpiper Calidris melanotos, Pallas's Gull Larus ichthyaetus, Lesser Crested Tern Thalasseus bengalensis, Black-winged Kite Elanus caeruleus, Rüppell's Warbler Sylvia rueppelli and Little Bunting Emberiza pusilla. Western Reef-egret Egretta gularis is not considered as a Little Egret Egretta garzetta subspecies anymore and is therefore included as a full species in the list of birds of Croatia. Two new subspecies have been accepted: Siberian subspecies of Common Stonechat Saxicola torquatus maurus and Eastern Great Egret Ardea alba modesta.

The Northern Bald Ibis Geronticus eremita, extinct in Croatia in the $17^{\text {th }}$ century (BAuer \& Glutz von Blotzheim 1996), have been observed on two occasions: an adult bird on 16 May 2016 in National Park Brijuni and a juvenile bird on 22 October 2016 near the mouth of river Mirna. Both birds had blue rings with white inscriptions on both legs (014 on the adult and 159 on the juvenile) pointing to the origin from Austrian/German reintroduced population. As this population is free-leaving but it is not clear if it is self-sustained, the Northern Bald Ibis is still retained in category $\mathrm{E}$ in Croatia.

Three species; Broad-billed Sandpiper Calidris falcinellus, Long-legged Buzzard Buteo rufinus and Pallid Harrier Circus macrourus are not listed as rare anymore because of the increased frequency of their observations. Furthermore, as Pallid Harrier occurrence throughout Western Europe and, particularly so, in France and the Iberian Peninsula have increased dramatically in the last decades, it has been suggested that a new migratory route is being established (DEL Hoyo et al. 2014), one that possibly partly crosses over Croatia (Corso \& CARdelli 2004; Terraube et al. 2012). Nonetheless, reporting observations of these species are still encouraged, but detailed descriptions of birds observed are not necessary anymore. Any indication of possible breeding attempt of Long-legged Buzzard in Croatia should also be reported as the species is breeding near the Croatian border, in Livanjsko polje in Bosnia and Herzegovina (Kotrošan \& Hatibović 2012).

Some bird taxa that would, based on the number of records, otherwise be excluded from rare bird taxa list are retained if their expected movement patterns are inconsistent with the appearance in Croatia (for example Paddyfield Warbler Acrocephalus agricola and Yellow-browed Warbler Phylloscopus inornatus). 


\section{CHANGES MADE COMPARED TO THE PREVIOUS REPORT}

Occurrence category Accidental (Ac) from previous report was lumped together with category Irregular (Ir), since bird watching in Croatia is neither intense enough nor subsequent observations numerous enough to make that kind of distinction. Consequently, all bird species that were previously awarded with category Ac are now put into category Ir.

Several species from the list of birds of Croatia are, after new assessment, included in the list of rare birds of Croatia. These species are Great Bustard Otis tarda, Rufous Scrub-robin Erythropygia galactotes, Aquatic Warbler Acrocephalus paludicola and Carrion Crow Corvus corone. Two species, Pallas's Leaf-warbler Phylloscopus proregulus and Red Phalarope Phalaropus fulicarius, that had previously been awarded category B as they were not observed in Croatia after 1950, are now awarded category A due to recent observation in Croatia. Owing to a recent split in species Common Redpoll Carduelis flammea into two species: Common Redpoll C. flammea and Lesser Redpoll C. cabaret, both species are for now treated as wintering birds in Croatia.

Two species are excluded from the list of birds of Croatia: Blyth's Reed-warbler, Acrocephalus dumetorum, due to erroneous inclusion, and Arctic Redpoll Carduelis hornemanni, due to insufficient confirmation. Six species: Red-legged Partridge Alectoris rufa, Muscovy Duck Cairina moschata, Wood Duck Aix sponsa, Yellow-billed Stork Mycteria ibis, Rose-ringed Parakeet Psittacula krameri and Yellow-crowned Bishop Euplectes afer had previously been erroneously awarded category D instead of category E and are now excluded from the list of birds of Croatia. Furthermore, species in category D are not included in the list of birds of Croatia anymore.

The list of rare bird taxa now includes 72 species, of which 5 are included in category C E (coming from self-sustained introduced populations but escapees could not be ruled out), and 4 subspecies. Eleven bird species are treated as escapees (category E) and one as probable escapee (category D).

The list of bird species of Croatia now contains 396 species in categories A, B and $C$ and 5 species in category $C$ E. Species in category D ( 1 species) and E (11 species) are not included in the list of the birds of Croatia (Appendix 1).

Abbreviations used:

ind. - individual

ad. - adult bird

imm. - immature bird

juv. - juvenile bird

$\widehat{0}$-male

†- female

is. - island 


\section{RARE BIRD TAXA LIST WITH ACCEPTED RECORDS IN THE PERIOD} 2012-2016.

\section{Category A}

1. White-headed Duck - Oxyura leucocephala - čakora - 2/2 25.04.2014, Neretva river mouth, 1 ad. 7, B. Ilić 18.05.2015, Varaždin lake, 1 ind., D. Bordjan (Figure 1)

2. Tundra Swan - Cygnus columbianus - mali labud - 5/11 08-09.12.2013, Polje Ozaljsko, 1 ad., T. Klanfar (Figure 2) 03.01.2014, Mirna river mouth, 1 ad., K. Mandić 15.11.2015, Draganić fishponds, 2 ad., T. Klanfar

3. Brent Goose - Branta bernicla - grivasta guska - 1/1

4. Barnacle Goose - Branta leucopsis - bjelolica guska - 1/1 13.01.2010, Podunavlje fishponds, 1 ind., A. Tomik

5. Red-breasted Goose - Branta ruficollis - crvenovrata guska - 11/37 12.01.2012, Lug, 7 ind., A. Tomik

12.01.2014, Pakra river accumulation, 1 ind., Ž. Vasilik

6. Pink-footed Goose - Anser brachyrhynchus - kratkokljuna guska - 4/9+

7. Lesser White-fronted Goose - Anser erythropus - mala guska - 2/11

8. Common Scoter - Melanitta nigra - crna patka - 9/14+ 16.12.2012, Neretva river mouth, 2 ad. $\hat{\sigma}+1$ ad. + , B. Ilić

9. Ruddy Shelduck - Tadorna ferruginea - zlatokrila utva - 4/6

10. Little Bustard - Tetrax tetrax - mala droplja - $1 / 1$

11. Great Bustard - Otis tarda - droplja - 4/20+

12. Common Loon - Gavia immer - veliki plijenor - $3 / 3$ 31.03.-06.04.2011, Rakitje accumulation, 1 juv., T. Rubinić 12.04.2012, Košljun bay, is. Pag, 1 ad., O. Samwald 17.-28.11.2015, Otok, Prelog, 1 juv., L. Hercigonja (Figure 3)

13. Yellow-billed Loon - Gavia adamsii - žutokljuni plijenor - $1 / 1$

14. European Storm-petrel - Hydrobates pelagicus - burnica - 1/2

15. Western Reef-egret - Egretta gularis - obalna čaplja - 1/1

16. Dalmatian Pelican - Pelecanus crispus - kudravi nesit - 5/6

17. Great White Pelican - Pelecanus onocrotalus - ružičasti nesit - 3/27

18. Northern Gannet - Morus bassanus - bluna - 2/2

19. American Golden Plover - Pluvialis dominica - američki zlatar - 1/1 28.04.2013, Dinjiška bay, is. Pag, 1 ind., A. Ploj (Figure 4)

20. Eurasian Dotterel - Eudromias morinellus - šareni kulik - 9/21+ 18.09.2012, Stara Baška, is. Krk, 6 ind., W. Hanschitz-Jandl 07.09.2015, Učka, 2 juv., B. Ječmenica 13.09.2015, Komiža, is. Vis, 1 juv., M. Šašić 15.-16.09.2015, Vrataruša, Senj, 3 ind., T. Klanfar (Figure 5) 
21. White-tailed Lapwing - Vanellus leucurus - bjelorepi vivak - $1 / 1$

22. Slender-billed Curlew - Numenius tenuirostris - tankokljuni pozviždač $1 / 1$

23. Broad-billed Sandpiper - Calidris falcinellus - plosnatokljuni žalar - 22/37 30.08.2012, Kolansko blato, is. Pag, 2 ind., A. Ploj

26-30.08.2013, Vrana lake, Pakoštane, 1 ad., T. Blažev and A. Ploj

30.08.2013, Dinjiška bay, is. Pag, 6 ind., A. Ploj

24.08.2016, Nin saltworks, 1 ind., J. Ledinščak

10.09.2016, Neretva river mouth, 1 juv., D. Dender

24. Pectoral Sandpiper - Calidris melanotos - žalar šljukar - 1/1

12.05.2012, Ploče (settling tank), 1 ad., I. Lolić (Figure 6)

25. Red-necked Phalarope - Phalaropus lobatus - tankokljuna liskonoga 7/10

26. Red Phalarope - Phalaropus fulicarius - riđa liskonoga - 1/1 06.05.2013, Blato, Zagreb, 1 ad. $\precsim$, Z. Tkalčec (Figure 7)

27. Terek Sandpiper - Xenus cinereus - prutka sabljarka - 3/3 30.08.2013, Dinjiška bay, is. Pag, 1 ad., A. Ploj (Figure 8)

28. Greater Yellowlegs - Tringa melanoleuca - žutonoga prutka - 1/1

29. Black-winged Pratincole - Glareola nordmanni - crnokrila pješčarka - $1 / 1$

30. Slender-billed Gull - Larus genei - tankokljuni galeb - 11/23

31. Franklin's Gull - Larus pipixcan - prerijski galeb - 1/1

32. Pallas's Gull - Larus ichthyaetus - veliki crnoglavi galeb - 1/1 13.03.2016, Jakuševec landfill, Zagreb, 1 ad., L. Jurinović (Figure 9)

33. Ring-billed Gull - Larus delawarensis - prstenokljuni galeb - 2/2

34. Great Black-backed Gull - Larus marinus - veliki galeb - 14/19 28.01.2012, Jakuševec landfill, Zagreb, 1 ad., Z. Tkalčec

26.01.2014, Jarun lake, Zagreb, 1 ad., M. Szymański (Figure 10)

11.12.2015, Jakuševec landfill, Zagreb, 3 ad., L. Jurinović and N. Brajdić

09.11.2016, Jakuševec landfill, Zagreb, 1 ad., L. Jurinović

35. Lesser Crested Tern - Thalasseus bengalensis - narančastokljuna čigra - 1/1

11.05.2014, Mirna river mouth, 1 ad., P. Tout (Figure 11)

36. Long-tailed Jaeger - Stercorarius longicaudus - dugorepi pomornik - $3 / 3$

37. Arctic Jaeger - Stercorarius parasiticus - kratkorepi pomornik - 8/9

15.12.2012, Blato, Zagreb, 1 ind., Z. Tkalčec

22.12.2012, Savica lakes, Zagreb, 1 juv., Ž. Kustec

38. Pomarine Jaeger - Stercorarius pomarinus - širokorepi pomornik - $1 / 1^{*}$

39. Atlantic Puffin - Fratercula arctica - tupik - 1/1 14.07.2015, Podaca, Gradac, 1 ad., N. Alač (Figure 12)

40. Black-winged Kite - Elanus caeruleus - crnokrila lunja - 1/1

28.08.-10.10.2016, Bokanjačko blato, Zadar, 1 ad., I. Dević and M. Stipčević (Figure 13) 
41. Pallid Harrier - Circus macrourus - stepska eja - 13/13 28.04.2013, Dinjiška bay, is. Pag, 1 imm. ô, A. Ploj 29.04.2013, Velo blato, is. Pag, 1 ad. + , A. Ploj 06.04.2014, Mahovo, 1 ad. $\widehat{\jmath}$, Ž. Vasilik 08.09.2014, Darda, 1 ad. $\widehat{\jmath}$, A. Tomik 25.03.2015, Konavosko polje, Konavle, 1 ad. đ̊, D. Dender 02.04.2015, Konavosko polje, Konavle, 1 ad. đَ, D. Dender 12.04.2016, Dinjiška, is. Pag, 1 imm., P. Tout 15.04.2016, is. Cres, 1 imm., R. Probst 16.04.2016, Imotski, 1 ad. đ̃, I. Lolić

42. Long-legged Buzzard - Buteo rufinus - riđi škanjac - 18/19 12.01.2013, Ježević, Vrlika, 1 ind., I. Budinski 21.04.2013, Imotsko polje, Imotski, 1 ind., I. Lolić 03.03.2014, Sinj, 1 ind., I. Lolić 31.03.2014, Vrana lake, Pakoštane, 1 ind., I. Lolić 22.06.2014, Donji Lapac, 1 ind., I. Lolić 17.01.2015, Ježevićko Suhopolje, Vrlika, 1 ind., I. Budinski 23.12.2015, Cetina, Vrlika, 1 ind., I. Budinski

43. Blue-cheeked Bee-eater - Merops persicus - plavolica pčelarica - $1 / 1$

44. Richard's Pipit - Anthus richardi - velika trepteljka - 2/2

45. Citrine Wagtail - Motacilla citreola - limunasta pastirica - 13/15 10.05.2011, Zapuntela, is. Molat, 1 ind., J. Mužinić and J. Purger 20.09.2013, Končanica fishponds, 1 ad. $\widehat{o}$, L. Jurinović 30.04.-01.05.2014, Kolansko blato, is. Pag, 1 ind., A. Ploj 23.04.2015, Velo Blato, is. Pag, 1 ad. đ̋, T. Rubinić (Figure 14) 07.04.2016, accumulation Ormož, 1 ad. $\widehat{O}$, B. Ječmenica, I. Šoštarić and S. Hodić

46. Black-throated Accentor - Prunella atrogularis - crnobradi popić - 1/1

47. Rufous Scrub-robin - Erythropygia galactotes - živičnjak - 1/1

48. Pied Wheatear - Oenanthe pleschanka - dvobojna bjeloguza - 1/1

49. Isabelline Wheatear - Oenanthe isabellina - azijska bjeloguza - $1 / 1$

50. Aquatic Warbler - Acrocephalus paludicola - trstenjak ševar - 2/2

51. Paddyfield Warbler - Acrocephalus agricola - poljski trstenjak - 19/19 29.09.2012, Vrana lake, Pakoštane, 1 juv., S. Barišić 03.10.2012, Velo Blato, is. Pag, 1 juv., D. Šere 23.08.2013, Vrana lake, Pakoštane, 1 juv., T. Blažev 28.08.2013, Vrana lake, Pakoštane, 1 juv., T. Blažev 14.08.2014, Vrana lake, Pakoštane, 1 juv., I. Lolić (Figure 15) 31.08.2014, Vrana lake, Pakoštane, 1 juv., T. Blažev 20.09.2014, Vrana lake, Pakoštane, 1 juv., I. Lolić 08.08.2015, Vid, Metković, 1 ad., B. Ilić 
31.08.2016, Vrana lake, Pakoštane, 1 ind., T. Blažev

52. Booted Warbler - Hippolais caligata - voljić žbunjar - 1/1

53. Pallas's Leaf-warbler - Phylloscopus proregulus - zlatoglavi zviždak - 1/1 01.11.2015, is. Unije, 1 ind., P. Corva (Figure 16)

54. Yellow-browed Warbler - Phylloscopus inornatus - azijski zviždak - 13/13 06.10.2014, Vid, Metković, 1 ind., B. Ilić 06.10.2014, Učka, 1 ind., P. Busse 19.04.2015, Mala Paklenica, 1 ad., G. Gorman 30.09.2015, Vid, Metković, 1 ind., B. Ilić 29.09.2016, Vrana lake, Pakoštane, 1 ind., S. Barišić 03.10.2016, Vrana lake, Pakoštane, 1 ind., S. Barišić

55. Hume's Leaf-warbler - Phylloscopus humei - srebrnastoprsi zviždak - 1/1

56. Rüppell's Warbler - Sylvia rueppelli - crnogrla grmuša - 1/1 05.05.2015, Cavtat, 1 ad. đ̋, D. Horton (Figure 17)

57. Carrion Crow - Corvus corone - crna vrana - $1 / 1$ 05.12.2015, Mirna river, 1 ind., P. Tout

58. White-winged Snowfinch - Montifringilla nivalis - snježni vrabac $-1 / 2$

59. Citril Finch - Carduelis citrinella - planinska žutarica - 3/15 22.11.2014, Vrataruša, Senj, 13 ind., T. Klanfar

60. Common Redpoll - Carduelis flammea - sjeverna juričica - $1 / 1$

61. Twite - Carduelis flavirostris - gorska juričica $-6 / 12$

62. Common Rosefinch - Carpodacus erythrinus - rujnica - 10/17

24.05.2010, is. Premuda, 2 ind., J. Mužinić and J. Purger 26.05.2010, Zapuntela, is. Molat, 4 ind., J. Mužinić and J. Purger 27.05.2010, Brgulje, is. Molat, 2 ind., J. Mužinić and J. Purger 24.04.2015, Vrhovine, Senj, 2 đ̊, T. Klanfar

63. Lapland Longspur - Calcarius lapponicus - laponska strnadica - 1/2

64. Snow Bunting - Plectrophenax nivalis - bijela strnadica - 7/8

07.12.2013, Predošćica, is. Cres, $1 \mathrm{imm}$. गै, E. Sos

29.11.2014, Neretva river mouth, 1 ad. đ̋, B. Ilić

27.12.2014, Blato, Zagreb, 2 ad. + + imm. ते, M. Szymański (Figure 18)

65. Pine Bunting - Emberiza leucocephalos - bjeloglava strnadica - 2/2

66. Little Bunting - Emberiza pusilla - mala strnadica - 1/1

30.10.1994, Mirna river, 1 ind., J. Dolinšek

67. Rustic Bunting - Emberiza rustica - šumska strnadica - 1/1

\section{Category C E}

Mandarin Duck - Aix galericulata - mandarinka - 8/9

06.11.2007, Dragonja river, $1++1 \delta$, W. Stani

07.01.2014, Jastrebarsko, $1++1 \hat{\jmath}$, A. Nevajda

Black Swan - Cygnus atratus - crni labud - 7/12 
Canada Goose - Branta canadensis - kanadska guska - 1/1

01.02.2013, Jelas polje, Slavonski Brod, 1 ind., N. Šetina

10.01.2016, Kupa near Sisak, 1 ad., Ž. Vasilik

Bar-headed Goose - Anser indicus - indijska guska - 2/2

Egyptian Goose - Alopochen aegyptiaca - egipatska guska - 4/4

24.05.2013, Darda, 1 ad., A. Tomik

06.07.-25.08.2013, Metković, 1 ad., B. Ilić

01.12.2015, Osijek, 1 ad., J. Ledinšćak

Category D

African Spoonbill - Platalea alba - afrička žličarka - 1/1

Category E

Barbary Partridge - Alectoris barbara - afrička jarebica - 1/1

04.05.2014, Vozilići, Plomin, 2 ind., A. Vrezec

Red-legged Partridge - Alectoris rufa - riđa jarebica - $1 / 1$

Muscovy Duck - Cairina moschata - mošusna patka - 1/1

Wood Duck - Aix sponsa - američka mandarinka - 8/9

White-cheeked Pintail - Anas bahamensis - smeđokapa patka - 1/1

07.04.2016, accumulation Ormož, 1 ind., B. Ječmenica, I. Šoštarić and S. Hodić

Chilean Flamingo - Phoenicopterus chilensis - čilski plamenac - 1/1

Yellow-billed Stork - Mycteria leucocephala - šarena roda - 1/1

Northern Bald Ibis - Geronticus eremita - ćelavi ibis - 2/2

16.05.2016, Veli Brijun, 1 ad., A. Richards

22.10.2016, Mirna river mouth; 1 juv., P. Tout

Lappet-faced Vulture - Torgos tracheliotus - veliki sup - 1/1

Rose-ringed Parakeet - Psittacula krameri - mala aleksandra - 1/1

Yellow-crowned Bishop - Euplectes afer - žutokruna pletilja-1/1

\section{SUBSPECIES}

Eastern Great Egret - Ardea alba modesta - 1/1

17.-18.11.2009, Kopački rit, 1 ind.

Black-eared Kite - Milvus migrans lineatus - azijska lunja - 1/1

Steppe Buzzard - Buteo buteo vulpinus - stepski škanjac - 2/2

Siberian Common Stonechat - Saxicola torquatus maurus - sibirski batić - 1/1

30.10.2011, Kolansko blato, is.Pag, 1 ind., D. Šere

\section{REJECTED RECORDS:}

Blyth's Reed-warbler - Acrocephalus dumetorum - trstenjak žbunjar

30.09.2009, Kolansko blato, is. Pag, 1 juv. 
Rock Pipit - Anthus petrosus - siva trepteljka

23.10.2014, Vid, Metković, 1 ind.

\section{ACKNOWLEDGEMENTS}

We are grateful to all observers who submitted records and photographs to the Croatian Birds Rarities Committee.

\section{References}

Bauer, K.M., Glutz von Blotzheim, U. (1996): Handbuch der Vögel Mitteleuropas Akademische Verlagsgesellschaft. Frankfurt.

Corso, A., CArdelli, C. (2004): The migration of Pallid Harrier across the central Mediterranean with particular reference to the Strait of Messina. British Birds 97(5): 238-246.

del Hoyo, J., Elliott, A., Sargatal, J., Christie, D.A., de Juana, E. (eds) (2014): Handbook of the Birds of the World Alive. Lynx Edicions, Barcelona.

Kotrošan, D., Hatibović, E. (2012): Raptors in Bosnia and Herzegovina - their status and perspectives for monitoring development. Acrocephalus 33(154-155): 173-179.

KRALJ, J., BARIŠIĆ, S. (2013): Rare birds in Croatia. Third report of the Croatian Rarities Committee. Natura Croatica 22(2): 375-396.

Mikuska, J., Mıкuska, T. (1994): Ptice Dunava na području Hrvatske. Anali Zavoda za znanstveni rad u Osijeku 10: 109-175.

Mikuska, J., Mikuska, T, Romulić, M. (2002): Ptice. Vodič kroz biološku raznolikost Kopačkog rita. Knjiga 1. JU PP Kopački rit.

Radović, D., Kralj, J., Sušić, G., Devidé, Z. (2005): Rječnik standardnih hrvatskih ptičjih naziva. I. dio. Nevrapčarke. Hrvatska akademija znanosti i umjetnosti, Zagreb.

Rubinič, B., Božıč, L., SACKL, P. (2002): The actual status of Slender-billed Gull Larus genei on the southern Dalmatian coast. Acrocephalus 23(110/111): 35-37.

Štumberger, B. (2005): Zalivski galeb Larus genei. Acrocephalus 26(124): 53.

Sušıć, G. (2015): Riđi škanjac Buteo rufinus. Priroda 105(1046): 45-47.

Terraube, J., Mougeot, F., Cornulier, T., Verma, A., Gavrilov, A., Arroyo, B. (2012): Broad wintering range and intercontinental migratory divide within a core population of the near-threatened pallid harrier: Migratory connectivity in central Asian pallid harriers. Diversity and Distributions 18(4): 401-409. 


\section{SAŽETAK}

Četvrti izvještaj Hrvatske komisije za rijetke vrste ptica donosi prihvaćena opažanja rijetkih vrsta i podvrsta ptica koja je komisija zaprimila u razdoblju 2012-2016. Deset vrsta ptica, od kojih je jedna odbjegla iz zatočeništva, te dvije podvrste u Hrvatskoj je zabilježeno po prvi put. Popis ptica Hrvatske sada sadrži 396 vrsta zabilježenih u kategorijama A, B i $C$ te 5 vrsta zabilježenih u kategoriji C E. Daljnjih 11 vrsta koje su zabilježene u kategoriji E i jedna u kategoriji D nisu uključene u popis ptica Hrvatske. Tri vrste su uklonjene s popisa rijetkih vrsta zbog povećane učestalosti njihovih opažanja u Hrvatskoj. Popis rijetkih vrsta sada obuhvaća 72 vrste i 4 podvrste u kategorijama A-D. 


\section{Appendix 1.}

The list of Bird taxa of Croatia (Category A, B, C)

Description of categories:

\section{AERC (Association of European Rarities Committees) (www.aerc.eu/guidelines.html):}

A Species that have been recorded in an apparently natural state at least once since 1st January 1950.

B Species that have been recorded in an apparently natural state but only between 1st January 1800 and 31st December 1949.

C Species that were originally introduced by man, but have established selfsupported breeding populations derived from introduced stock, also birds probably originating from a category $\mathrm{C}$ population in other country.

D Species that would otherwise appear in categories A or B except that there is reasonable doubt that they have ever occurred in a natural state.

E Species that have been recorded as escapees from captivity.

\section{Occurrence}

$\mathrm{R}$ Regular - species recorded in at least 7 years out of the last 10 years

Ir Irregular - species recorded more than 10 times and in more than 5 years in the last 50 years but in fewer than 7 years out of the last 10 years

Rare - species recorded 1-10 times or in 1-5 years in the last 50 years

\section{Season}

B Breeding species

W Wintering species

M Migratory species

Re- extinct in Croatia in associated status

* species is irregular in the associated status

\begin{tabular}{|c|c|c|c|c|c|c|}
\hline & English name & Scientific name & Croatian name & $\begin{array}{c}\text { Category } \\
\text { (AERC) }\end{array}$ & Occurrence & Season \\
\hline 1. & Northern Bobwhite & Colinus virginianus & virdžinijska prepelica & $\mathrm{C}$ & $\mathrm{R}$ & B \\
\hline 2 . & Common Quail & Coturnix coturnix & prepelica & A & $\mathrm{R}$ & $\mathrm{B}, \mathrm{M}, \mathrm{W}^{*}$ \\
\hline 3. & Rock Partridge & Alectoris graeca & jarebica kamenjarka & A & $\mathrm{R}$ & B \\
\hline 4. & Chukar & Alectoris chukar & čukara & $\mathrm{C}$ & $\mathrm{R}$ & B \\
\hline 5 & Common Pheasant & Phasianus colchicus & fazan & $\mathrm{C}$ & $\mathrm{R}$ & B \\
\hline
\end{tabular}




\begin{tabular}{|c|c|c|c|c|c|c|}
\hline & English name & Scientific name & Croatian name & $\begin{array}{l}\text { Category } \\
\text { (AERC) }\end{array}$ & Occurrence & Season \\
\hline 6. & Grey Partridge & Perdix perdix & trčka & $\mathrm{A}$ & $\mathrm{R}$ & B \\
\hline 7. & Hazel Grouse & Bonasa bonasia & lještarka & $A$ & $\mathrm{R}$ & B \\
\hline 8. & Western Capercaillie & Tetrao urogallus & tetrijeb gluhan & A & $\mathrm{R}$ & B \\
\hline 9. & Black Grouse & Lyrurus tetrix & tetrijeb ruševac & B & & $\begin{array}{l}\text { M-Re, } \\
\text { W-Re }\end{array}$ \\
\hline 10. & White-headed Duck & Oxyura leucocephala & čakora & A & Rare & W-Re \\
\hline 11. & Black Swan & Cygnus atratus & crni labud & $\mathrm{CE}$ & Rare & \\
\hline 12. & Mute Swan & Cygnus olor & crvenokljuni labud & $A$ & $\mathrm{R}$ & $B, W$ \\
\hline 13. & Whooper Swan & Cygnus cygnus & žutokljuni labud & A & $\operatorname{Ir}$ & $\mathrm{W}^{*}$ \\
\hline 14. & Tundra Swan & Cygnus columbianus & mali labud & A & Rare & \\
\hline 15. & Brent Goose & Branta bernicla & grivasta guska & A & Rare & \\
\hline 16. & Barnacle Goose & Branta leucopsis & bjelolica guska & A & Rare & \\
\hline 17. & Red-breasted Goose & Branta ruficollis & crvenovrata guska & A & Rare & \\
\hline 18. & Canada Goose & Branta canadensis & kanadska guska & $C E$ & Rare & \\
\hline 19. & Bar-headed Goose & Anser indicus & indijska guska & $C E$ & Rare & \\
\hline 20. & Greylag Goose & Anser anser & siva guska & A & $\mathrm{R}$ & $B, M, W$ \\
\hline 21. & Bean Goose & Anser fabalis & guska glogovnjača & A & $\mathrm{R}$ & W \\
\hline 22. & Pink-footed Goose & Anser brachyrhynchus & kratkokljuna guska & A & Rare & \\
\hline 23. & $\begin{array}{l}\text { Greater White- } \\
\text { fronted Goose }\end{array}$ & Anser albifrons & lisasta guska & A & $\mathrm{R}$ & $\mathrm{M}, \mathrm{W}$ \\
\hline 24. & $\begin{array}{l}\text { Lesser White-fronted } \\
\text { Goose }\end{array}$ & Anser erythropus & mala guska & A & Rare & \\
\hline 25. & Long-tailed Duck & Clangula hyemalis & patka ledara & A & Ir & \\
\hline 26. & Common Eider & Somateria mollissima & gavka & A & Ir & \\
\hline 27. & Velvet Scoter & Melanitta fusca & patka kulašica & A & Ir & $W^{*}$ \\
\hline 28. & Common Scoter & Melanitta nigra & crna patka & A & Rare & \\
\hline 29. & Common Goldeneye & Bucephala clangula & patka batoglavica & A & $\mathrm{R}$ & W \\
\hline 30. & Smew & Mergellus albellus & bijeli ronac & A & $\mathrm{R}$ & W \\
\hline 31. & Goosander & Mergus merganser & veliki ronac & $\mathrm{A}$ & $\mathrm{R}$ & $B, W$ \\
\hline 32. & $\begin{array}{l}\text { Red-breasted } \\
\text { Merganser }\end{array}$ & Mergus serrator & mali ronac & $\mathrm{A}$ & $\mathrm{R}$ & W \\
\hline 33. & Egyptian Goose & Alopochen aegyptiaca & egipatska guska & $\mathrm{CE}$ & Rare & \\
\hline 34. & Common Shelduck & Tadorna tadorna & utva & A & Ir & $B^{*}, M^{*}, W^{*}$ \\
\hline 35. & Ruddy Shelduck & Tadorna ferruginea & zlatokrila utva & A & Rare & \\
\hline 36. & Mandarin Duck & Aix galericulata & mandarinka & $C E$ & Rare & \\
\hline 37. & Red-crested Pochard & Netta rufina & patka gogoljica & A & $\mathrm{R}$ & $B, M, W$ \\
\hline 38. & Common Pochard & Aythya ferina & glavata patka & A & $\mathrm{R}$ & $B, M, W$ \\
\hline 39. & Ferruginous Duck & Aythya nyroca & patka njorka & A & $\mathrm{R}$ & $B, M, W$ \\
\hline 40. & Tufted Duck & Aythya fuligula & krunata patka & A & $\mathrm{R}$ & $B, M, W$ \\
\hline 41. & Greater Scaup & Aythya marila & patka crninka & A & $\operatorname{Ir}$ & $\mathrm{M}^{*}, \mathrm{~W}^{*}$ \\
\hline
\end{tabular}




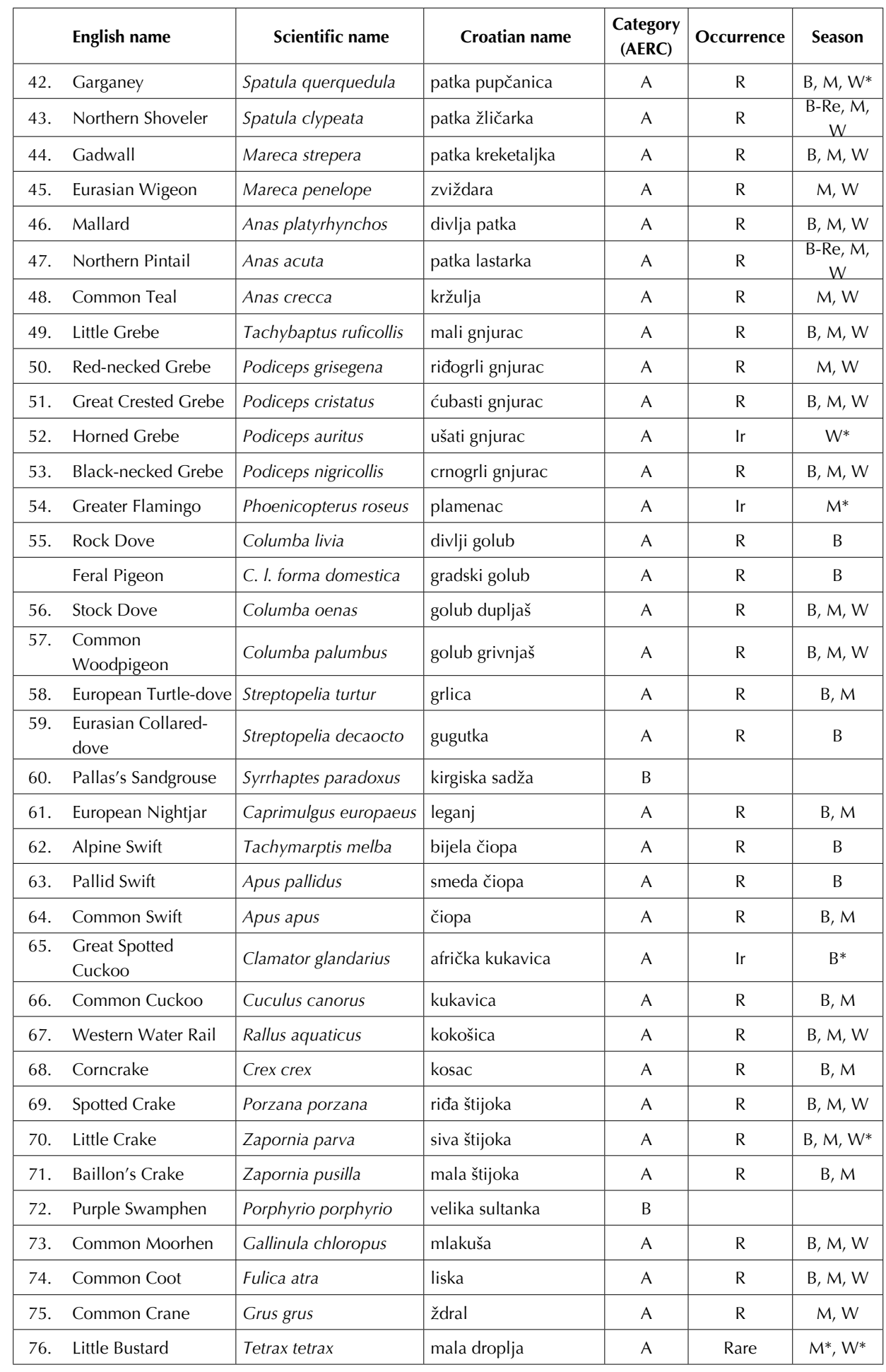




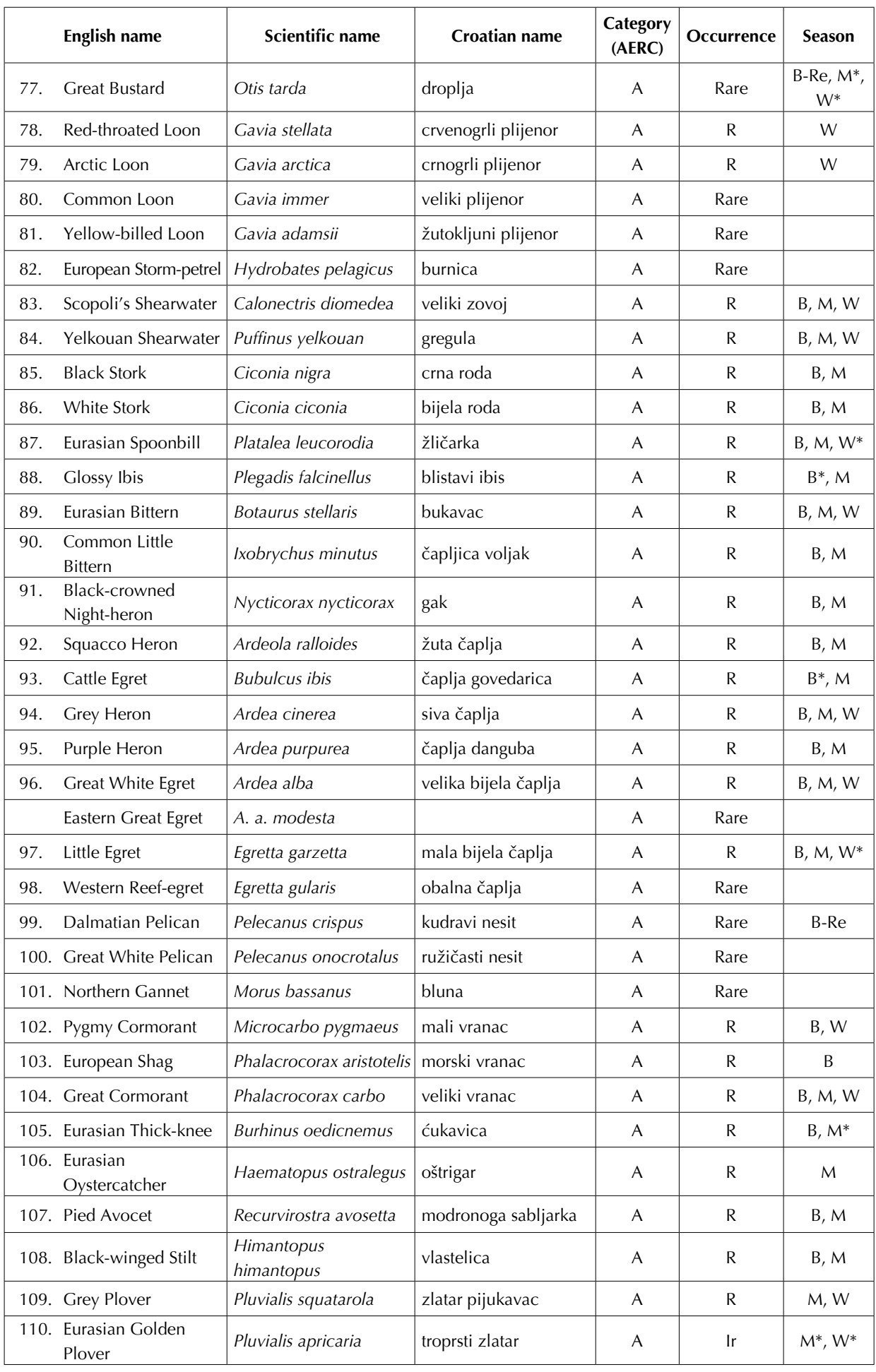




\begin{tabular}{|c|c|c|c|c|c|}
\hline English name & Scientific name & Croatian name & $\begin{array}{l}\text { Category } \\
\text { (AERC) }\end{array}$ & Occurrence & Season \\
\hline $\begin{array}{l}\text { 111. American Golden } \\
\text { Plover }\end{array}$ & Pluvialis dominica & američki zlatar & A & Rare & \\
\hline 112. Eurasian Dotterel & Eudromias morinellus & šareni kulik & A & Rare & \\
\hline $\begin{array}{l}\text { 113. Common Ringed } \\
\text { Plover }\end{array}$ & Charadrius hiaticula & kulik blatarić & A & $\mathrm{R}$ & $M, W^{*}$ \\
\hline 114. Little Ringed Plover & Charadrius dubius & kulik sljepčić & A & $\mathrm{R}$ & $B, M$ \\
\hline 115. Kentish Plover & Charadrius alexandrinus & morski kulik & A & $\mathrm{R}$ & $B, M^{*}$ \\
\hline 116. Northern Lapwing & Vanellus vanellus & vivak & A & $\mathrm{R}$ & $B, M, W$ \\
\hline $\begin{array}{l}\text { 117. Spur-winged } \\
\text { Lapwing }\end{array}$ & Vanellus spinosus & ostrugasti vivak & B & & \\
\hline 118. White-tailed Lapwing & Vanellus leucurus & bjelorepi vivak & A & Rare & \\
\hline 119. Upland Sandpiper & Bartramia longicauda & prerijska prutka & $\mathrm{B}$ & & \\
\hline 120. Whimbrel & Numenius phaeopus & prugasti pozviždač & A & $\mathrm{R}$ & M \\
\hline $\begin{array}{l}\text { 121. Slender-billed } \\
\text { Curlew }\end{array}$ & Numenius tenuirostris & tankokljuni pozviždač & A & Rare & $M^{*}$ \\
\hline 122. Eurasian Curlew & Numenius arquata & veliki pozviždač & A & $\mathrm{R}$ & $\mathrm{M}, \mathrm{W}$ \\
\hline 123. Bar-tailed Godwit & Limosa lapponica & riđa muljača & A & Ir & $M^{*}, W^{*}$ \\
\hline 124. Black-tailed Godwit & Limosa limosa & crnorepa muljača & A & $\mathrm{R}$ & M \\
\hline 125. Ruddy Turnstone & Arenaria interpres & kameničar & A & $\operatorname{Ir}$ & $M^{*}, W^{*}$ \\
\hline 126. Red Knot & Calidris canutus & rđasti žalar & A & Ir & $M^{*}$ \\
\hline 127. Ruff & Calidris pugnax & pršljivac & A & $\mathrm{R}$ & $M, W^{*}$ \\
\hline $\begin{array}{l}\text { 128. Broad-billed } \\
\text { Sandpiper }\end{array}$ & Calidris falcinellus & plosnatokljuni žalar & A & Rare & $M^{*}$ \\
\hline 129. Curlew Sandpiper & Calidris ferruginea & krivokljuni žalar & A & $\mathrm{R}$ & M \\
\hline 130. Temminck's Stint & Calidris temminckii & sijedi žalar & A & $\mathrm{R}$ & M \\
\hline 131. Sanderling & Calidris alba & bijeli žalar & A & $\mathrm{R}$ & M \\
\hline 132. Dunlin & Calidris alpina & žalar cirikavac & A & $\mathrm{R}$ & $M, W$ \\
\hline 133. Purple Sandpiper & Calidris maritima & morski žalar & $\mathrm{B}$ & & \\
\hline 134. Little Stint & Calidris minuta & mali žalar & A & $\mathrm{R}$ & M \\
\hline 135. Pectoral Sandpiper & Calidris melanotos & žalar šljukar & A & Rare & \\
\hline 136. Eurasian Woodcock & Scolopax rusticola & šljuka & A & $\mathrm{R}$ & $B, M, W$ \\
\hline 137. Great Snipe & Gallinago media & šljuka livadarka & A & Ir & $M^{*}$ \\
\hline 138. Common Snipe & Gallinago gallinago & šljuka kokošica & A & $\mathrm{R}$ & $B, M, W$ \\
\hline 139. Jack Snipe & Lymnocryptes minimus & mala šljuka & A & $\mathrm{R}$ & $\mathrm{M}, \mathrm{W}$ \\
\hline $\begin{array}{l}\text { 140. } \text { Red-necked } \\
\text { Phalarope }\end{array}$ & Phalaropus lobatus & tankokljuna liskonoga & A & Rare & \\
\hline 141. Red Phalarope & Phalaropus fulicarius & riđa liskonoga & A & Rare & \\
\hline 142. Terek Sandpiper & Xenus cinereus & prutka sabljarka & A & Rare & \\
\hline 143. Common Sandpiper & Actitis hypoleucos & mala prutka & A & $\mathrm{R}$ & $B, M, W$ \\
\hline 144. Green Sandpiper & Tringa ochropus & crnokrila prutka & A & $\mathrm{R}$ & $M, W$ \\
\hline 145. Spotted Redshank & Tringa erythropus & crna prutka & A & $\mathrm{R}$ & M \\
\hline
\end{tabular}




\begin{tabular}{|c|c|c|c|c|c|}
\hline English name & Scientific name & Croatian name & $\begin{array}{l}\text { Category } \\
\text { (AERC) }\end{array}$ & Occurrence & Season \\
\hline $\begin{array}{l}\text { 146. Common } \\
\text { Greenshank }\end{array}$ & Tringa nebularia & krivokljuna prutka & A & $\mathrm{R}$ & $M, W^{*}$ \\
\hline 147. Greater Yellowlegs & Tringa melanoleuca & žutonoga prutka & A & Rare & \\
\hline 148. Common Redshank & Tringa totanus & crvenonoga prutka & A & $\mathrm{R}$ & B, M, W \\
\hline 149. Wood Sandpiper & Tringa glareola & prutka migavica & A & $\mathrm{R}$ & M \\
\hline 150. Marsh Sandpiper & Tringa stagnatilis & dugonoga prutka & $A$ & $\mathrm{R}$ & M \\
\hline $\begin{array}{l}\text { 151. Cream-coloured } \\
\text { Courser }\end{array}$ & Cursorius cursor & smeđa trkalica & B & & \\
\hline 152. Collared Pratincole & Glareola pratincola & riđokrila pješčarka & A & Ir & $M^{*}$ \\
\hline $\begin{array}{l}\text { 153. Black-winged } \\
\text { Pratincole }\end{array}$ & Glareola nordmanni & crnokrila pješčarka & A & Rare & \\
\hline 154. Little Gull & Hydrocoloeus minutus & mali galeb & A & $\mathrm{R}$ & $M, W$ \\
\hline $\begin{array}{l}\text { 155. Black-legged } \\
\text { Kittiwake }\end{array}$ & Rissa tridactyla & troprsti galeb & A & Ir & \\
\hline 156. Slender-billed Gull & Larus genei & tankokljuni galeb & A & Rare & \\
\hline 157. Black-headed Gull & Larus ridibundus & riječni galeb & A & $\mathrm{R}$ & $B, M, W$ \\
\hline 158. Franklin's Gull & Larus pipixcan & prerijski galeb & A & Rare & \\
\hline 159. Pallas's Gull & Larus ichthyaetus & veliki crnoglavi galeb & A & Rare & \\
\hline 160. Mediterranean Gull & Larus melanocephalus & crnoglavi galeb & A & $\mathrm{R}$ & M \\
\hline 161. Audouin's Gull & Larus audouinii & sredozemni galeb & A & $\mathrm{R}$ & B \\
\hline 162. Ring-billed Gull & Larus delawarensis & prstenokljuni galeb & A & Rare & \\
\hline 163. Mew Gull & Larus canus & burni galeb & A & $\mathrm{R}$ & $M, W$ \\
\hline $\begin{array}{l}\text { 164. Lesser Black-backed } \\
\text { Gull }\end{array}$ & Larus fuscus & tamnoleđi galeb & A & $\mathrm{R}$ & $M, W$ \\
\hline $\begin{array}{l}\text { 165. European Herring } \\
\text { Gull } \\
\end{array}$ & Larus argentatus & srebrnasti galeb & A & $\mathrm{R}$ & $M, W$ \\
\hline 166. Yellow-legged Gull & Larus michahellis & galeb klaukavac & A & $\mathrm{R}$ & $B, M, W$ \\
\hline 167. Caspian Gull & Larus cachinnans & pontski galeb & A & $\mathrm{R}$ & $M, W$ \\
\hline $\begin{array}{l}\text { 168. Great Black-backed } \\
\text { Gull }\end{array}$ & Larus marinus & veliki galeb & A & Rare & \\
\hline 169. Little Tern & Sternula albifrons & mala čigra & A & $\mathrm{R}$ & $B, M$ \\
\hline $\begin{array}{l}\text { 170. Common Gull-billed } \\
\text { Tern }\end{array}$ & Gelochelidon nilotica & debelokljuna čigra & A & Ir & $B-\operatorname{Re}^{*}, M^{*}$ \\
\hline 171. Caspian Tern & Hydroprogne caspia & velika čigra & A & $\mathrm{R}$ & M \\
\hline 172. Whiskered Tern & Chlidonias hybrida & bjelobrada čigra & A & $\mathrm{R}$ & $B, M$ \\
\hline 173. White-winged Tern & Chlidonias leucopterus & bjelokrila čigra & A & $\mathrm{R}$ & B-Re*, M \\
\hline 174. Black Tern & Chlidonias niger & crna čigra & A & $\mathrm{R}$ & B-Re*, M \\
\hline 175. Common Tern & Sterna hirundo & crvenokljuna čigra & A & $\mathrm{R}$ & $B, M$ \\
\hline 176. Lesser Crested Tern & Thalasseus bengalensis & narančastokljuna čigra & A & Rare & \\
\hline 177. Sandwich Tern & Thalasseus sandvicensis & dugokljuna čigra & A & $\mathrm{R}$ & $\mathrm{M}, \mathrm{W}$ \\
\hline 178. Long-tailed Jaeger & $\begin{array}{l}\text { Stercorarius } \\
\text { longicaudus }\end{array}$ & dugorepi pomornik & A & Rare & \\
\hline 179. Arctic Jaeger & Stercorarius parasiticus & kratkorepi pomornik & A & Rare & \\
\hline
\end{tabular}




\begin{tabular}{|c|c|c|c|c|c|}
\hline English name & Scientific name & Croatian name & $\begin{array}{c}\text { Category } \\
\text { (AERC) }\end{array}$ & Occurrence & Season \\
\hline 180. Pomarine Jaeger & Stercorarius pomarinus & širokorepi pomornik & A & Rare & \\
\hline 181. Great Skua & Catharacta skua & veliki pomornik & B & & \\
\hline 182. Atlantic Puffin & Fratercula arctica & tupik & A & Rare & \\
\hline 183. Black Guillemot & Cepphus grylle & crna njorka & B & & \\
\hline 184. Razorbill & Alca torda & plosnatokljuna njorka & B & & \\
\hline 185. Common Barn-owl & Tyto alba & kukuvija & A & $\mathrm{R}$ & B \\
\hline 186. Eurasian Pygmy-owl & Glaucidium passerinum & mali ćuk & A & $\mathrm{R}$ & B \\
\hline 187. Little Owl & Athene noctua & sivi ćuk & A & $\mathrm{R}$ & B \\
\hline 188. Boreal Owl & Aegolius funereus & planinski ćuk & A & $\mathrm{R}$ & $\mathrm{B}$ \\
\hline 189. Eurasian Scops-owl & Otus scops & ćuk & A & $\mathrm{R}$ & $B, M$ \\
\hline $\begin{array}{l}\text { 190. Northern Long-eared } \\
\text { Owl }\end{array}$ & Asio otus & mala ušara & A & $\mathrm{R}$ & $\mathrm{B}, \mathrm{M}, \mathrm{W}$ \\
\hline 191. Short-eared Owl & Asio flammeus & sova močvarica & A & $\mathrm{R}$ & $B^{*}, M^{*}, W$ \\
\hline 192. Tawny Owl & Strix aluco & šumska sova & A & $\mathrm{R}$ & B \\
\hline 193. Ural Owl & Strix uralensis & jastrebača & A & $\mathrm{R}$ & $\mathrm{B}$ \\
\hline 194. Eurasian Eagle-owl & Bubo bubo & ušara & A & $\mathrm{R}$ & B \\
\hline 195. Osprey & Pandion haliaetus & bukoč & A & $\mathrm{R}$ & B-Re, M \\
\hline 196. Black-winged Kite & Elanus caeruleus & crnokrila lunja & A & Rare & \\
\hline $\begin{array}{l}\text { 197. European Honey- } \\
\text { buzzard }\end{array}$ & Pernis apivorus & škanjac osaš & A & $\mathrm{R}$ & $\mathrm{B}, \mathrm{M}$ \\
\hline 198. Bearded Vulture & Gypaetus barbatus & kostoberina & $\mathrm{B}$ & & \\
\hline 199. Egyptian Vulture & Neophron percnopterus & crkavica & A & & B-Re \\
\hline $\begin{array}{l}\text { 200. Short-toed Snake- } \\
\text { eagle }\end{array}$ & Circaetus gallicus & zmijar & A & $\mathrm{R}$ & B \\
\hline 201. Griffon Vulture & Gyps fulvus & bjeloglavi sup & A & $\mathrm{R}$ & $\mathrm{B}$ \\
\hline 202. Cinereous Vulture & Aegypius monachus & crni lešinar & $\mathrm{B}$ & & $\mathrm{B}-\mathrm{Re}$ \\
\hline 203. Lesser Spotted Eagle & Clanga pomarina & orao kliktaš & A & $\mathrm{R}$ & B \\
\hline 204. Greater Spotted Eagle & Clanga clanga & orao klokotaš & A & $\mathrm{R}$ & W \\
\hline 205. Steppe Eagle & Aquila nipalensis & stepski orao & B & & \\
\hline $\begin{array}{l}\text { 206. Eastern Imperial } \\
\text { Eagle }\end{array}$ & Aquila heliaca & orao krstaš & A & $\mathrm{R}$ & B \\
\hline 207. Golden Eagle & Aquila chrysaetos & suri orao & A & $\mathrm{R}$ & $\mathrm{B}$ \\
\hline 208. Bonelli's Eagle & Aquila fasciata & prugasti orao & A & $\mathrm{R}$ & B \\
\hline 209. Booted Eagle & Hieraaetus pennatus & patuljasti orao & A & $\mathrm{R}$ & $\mathrm{B}, \mathrm{M}^{*}$ \\
\hline $\begin{array}{l}\text { 210. Western Marsh- } \\
\text { harrier }\end{array}$ & Circus aeruginosus & eja močvarica & A & $\mathrm{R}$ & $B, M, W$ \\
\hline 211. Hen Harrier & Circus cyaneus & eja strnjarica & A & $\mathrm{R}$ & $M, W$ \\
\hline 212. Pallid Harrier & Circus macrourus & stepska eja & A & Rare & \\
\hline 213. Montagu's Harrier & Circus pygargus & eja livadarka & A & $\mathrm{R}$ & $\mathrm{B}, \mathrm{M}, \mathrm{W}^{*}$ \\
\hline 214. Levant Sparrowhawk & Accipiter brevipes & kratkoprsti kobac & A & $\mathrm{R}$ & B \\
\hline
\end{tabular}




\begin{tabular}{|c|c|c|c|c|c|}
\hline English name & Scientific name & Croatian name & $\begin{array}{l}\text { Category } \\
\text { (AERC) }\end{array}$ & Occurrence & Season \\
\hline $\begin{array}{l}\text { 215. Eurasian } \\
\text { Sparrowhawk }\end{array}$ & Accipiter nisus & kobac & A & $\mathrm{R}$ & $B, M, W$ \\
\hline 216. Northern Goshawk & Accipiter gentilis & jastreb & A & $\mathrm{R}$ & $\mathrm{B}, \mathrm{W}^{*}$ \\
\hline $\begin{array}{l}\text { 217. White-tailed Sea- } \\
\text { eagle }\end{array}$ & Haliaeetus albicilla & štekavac & A & $\mathrm{R}$ & B \\
\hline 218. Red Kite & Milvus milvus & crvena lunja & A & $R$ & $\begin{array}{c}\mathrm{B}-\mathrm{Re}, \mathrm{M}^{*}, \\
\mathrm{~W}^{*}\end{array}$ \\
\hline 219. Black Kite & Milvus migrans & crna lunja & A & $\mathrm{R}$ & B, M \\
\hline Black-eared Kite & M. m. lineatus & azijska lunja & A & Rare & \\
\hline $\begin{array}{l}\text { 220. Rough-legged } \\
\text { Buzzard }\end{array}$ & Buteo lagopus & škanjac gaćaš & A & Ir & $W^{*}$ \\
\hline 221. Eurasian Buzzard & Buteo buteo & škanjac & A & $\mathrm{R}$ & $B, M, W$ \\
\hline Steppe Buzzard & B. b. vulpinus & stepski škanjac & A & Rare & \\
\hline 222. Long-legged Buzzard & Buteo rufinus & riđi škanjac & A & Rare & \\
\hline 223. Common Hoopoe & Upupa epops & pupavac & A & $\mathrm{R}$ & $B, M$ \\
\hline $\begin{array}{l}\text { 224. Blue-cheeked Bee- } \\
\text { eater }\end{array}$ & Merops persicus & plavolica pčelarica & A & Rare & \\
\hline 225. European Bee-eater & Merops apiaster & pčelarica & A & $\mathrm{R}$ & B, M \\
\hline 226. European Roller & Coracias garrulus & zlatovrana & A & $\mathrm{R}$ & $B, M$ \\
\hline 227. Common Kingfisher & Alcedo atthis & vodomar & A & $\mathrm{R}$ & $B, M, W$ \\
\hline 228. Eurasian Wryneck & Jynx torquilla & vijoglav & A & $\mathrm{R}$ & $B, M$ \\
\hline $\begin{array}{l}\text { 229. Grey-faced } \\
\text { Woodpecker }\end{array}$ & Picus canus & siva žuna & A & $\mathrm{R}$ & B \\
\hline $\begin{array}{l}\text { 230. Eurasian Green } \\
\text { Woodpecker }\end{array}$ & Picus viridis & zelena žuna & A & $\mathrm{R}$ & B \\
\hline 231. Black Woodpecker & Dryocopus martius & crna žuna & A & $\mathrm{R}$ & B \\
\hline $\begin{array}{l}\text { 232. } \text { Three-toed } \\
\text { Woodpecker }\end{array}$ & Picoides tridactylus & troprsti djetlić & A & $\mathrm{R}$ & B \\
\hline $\begin{array}{l}\text { 233. Middle Spotted } \\
\text { Woodpecker }\end{array}$ & Leiopicus medius & crvenoglavi djetlić & A & $\mathrm{R}$ & B \\
\hline $\begin{array}{l}\text { 234. Lesser Spotted } \\
\text { Woodpecker }\end{array}$ & Dryobates minor & mali djetlić & A & $\mathrm{R}$ & B \\
\hline $\begin{array}{l}\text { 235. White-backed } \\
\text { Woodpecker }\end{array}$ & Dendrocopos leucotos & planinski djetlić & A & $\mathrm{R}$ & B \\
\hline 236. Syrian Woodpecker & Dendrocopos syriacus & sirijski djetlić & A & $\mathrm{R}$ & B \\
\hline $\begin{array}{l}\text { 237. Great Spotted } \\
\text { Woodpecker }\end{array}$ & Dendrocopos major & veliki djetlić & A & $\mathrm{R}$ & B \\
\hline 238. Lesser Kestrel & Falco naumanni & bjelonokta vjetruša & A & $\mathrm{R}$ & $\mathrm{B}, \mathrm{M}$ \\
\hline 239. Common Kestrel & Falco tinnunculus & vjetruša & A & $\mathrm{R}$ & $B, M, W$ \\
\hline 240. Red-footed Falcon & Falco vespertinus & crvenonoga vjetruša & A & $\mathrm{R}$ & M \\
\hline 241. Eleonora's Falcon & Falco eleonorae & Eleonorin sokol & A & $\mathrm{R}$ & $\mathrm{B}$ \\
\hline 242. Merlin & Falco columbarius & mali sokol & A & $\mathrm{R}$ & $M, W$ \\
\hline 243. Eurasian Hobby & Falco subbuteo & sokol lastavičar & A & $\mathrm{R}$ & $B, M$ \\
\hline 244. Lanner Falcon & Falco biarmicus & krški sokol & A & $\mathrm{R}$ & B \\
\hline
\end{tabular}




\begin{tabular}{|c|c|c|c|c|c|}
\hline English name & Scientific name & Croatian name & $\begin{array}{l}\text { Category } \\
\text { (AERC) }\end{array}$ & Occurrence & Season \\
\hline 245. Saker Falcon & Falco cherrug & stepski sokol & A & $\mathrm{R}$ & $\mathrm{B}, \mathrm{M}, \mathrm{W}^{*}$ \\
\hline 246. Peregrine Falcon & Falco peregrinus & sivi sokol & A & $\mathrm{R}$ & $B, M^{*}, W$ \\
\hline 247. Calandra Lark & $\begin{array}{l}\text { Melanocorypha } \\
\text { calandra }\end{array}$ & velika ševa & A & $\mathrm{R}$ & $\mathrm{B}$ \\
\hline $\begin{array}{l}\text { 248. Greater Short-toed } \\
\text { Lark }\end{array}$ & $\begin{array}{l}\text { Calandrella } \\
\text { brachydactyla }\end{array}$ & kratkoprsta ševa & A & $\mathrm{R}$ & B \\
\hline 249. Horned Lark & Eremophila alpestris & planinska ševa & A & $\mathrm{R}$ & B \\
\hline 250. Crested Lark & Galerida cristata & kukmasta ševa & A & $\mathrm{R}$ & B \\
\hline 251. Eurasian Skylark & Alauda arvensis & poljska ševa & A & $\mathrm{R}$ & $B, M, W$ \\
\hline 252. Woodlark & Lullula arborea & ševa krunica & A & $\mathrm{R}$ & $B, M, W$ \\
\hline 253. Collared Sand Martin & Riparia riparia & bregunica & A & $\mathrm{R}$ & B, M \\
\hline 254. Barn Swallow & Hirundo rustica & lastavica & A & $\mathrm{R}$ & $B, M$ \\
\hline 255. Eurasian Crag Martin & Ptyonoprogne rupestris & hridna lastavica & A & $\mathrm{R}$ & $\mathrm{B}$ \\
\hline $\begin{array}{l}\text { 256. Northern House } \\
\text { Martin }\end{array}$ & Delichon urbicum & piljak & A & $\mathrm{R}$ & $B, M$ \\
\hline 257. Red-rumped Swallow & Cecropis daurica & daurska lastavica & A & $\mathrm{R}$ & B \\
\hline 258. Richard's Pipit & Anthus richardi & velika trepteljka & A & Rare & \\
\hline 259. Tawny Pipit & Anthus campestris & primorska trepteljka & A & $\mathrm{R}$ & $B, M$ \\
\hline 260. Meadow Pipit & Anthus pratensis & livadna trepteljka & A & $\mathrm{R}$ & $M, W$ \\
\hline 261. Red-throated Pipit & Anthus cervinus & rusogrla trepteljka & A & Ir & $M^{*}$ \\
\hline 262. Tree Pipit & Anthus trivialis & prugasta trepteljka & A & $\mathrm{R}$ & $B, M$ \\
\hline 263. Water Pipit & Anthus spinoletta & planinska trepteljka & A & $\mathrm{R}$ & $B, M, W$ \\
\hline 264. White Wagtail & Motacilla alba & bijela pastirica & A & $\mathrm{R}$ & $\mathrm{B}, \mathrm{M}, \mathrm{W}$ \\
\hline 265. Yellow Wagtail & Motacilla flava & žuta pastirica & A & $\mathrm{R}$ & $B, M$ \\
\hline 266. Citrine Wagtail & Motacilla citreola & limunasta pastirica & A & Rare & \\
\hline 267. Grey Wagtail & Motacilla cinerea & gorska pastirica & A & $\mathrm{R}$ & $B, M, W$ \\
\hline 268. Bohemian Waxwing & Bombycilla garrulus & kugara & A & Ir & $W^{*}$ \\
\hline $\begin{array}{l}\text { 269. White-throated } \\
\text { Dipper }\end{array}$ & Cinclus cinclus & vodenkos & A & $\mathrm{R}$ & B \\
\hline 270. Northern Wren & Troglodytes troglodytes & palčić & A & $\mathrm{R}$ & $B, M, W$ \\
\hline 271. Alpine Accentor & Prunella collaris & alpski popić & A & $\mathrm{R}$ & $\mathrm{B}$ \\
\hline $\begin{array}{l}\text { 272. Black-throated } \\
\text { Accentor }\end{array}$ & Prunella atrogularis & crnobradi popić & A & Rare & \\
\hline 273. Dunnock & Prunella modularis & sivi popić & A & $\mathrm{R}$ & $\mathrm{B}, \mathrm{M}, \mathrm{W}$ \\
\hline 274. Mistle Thrush & Turdus viscivorus & drozd imelaš & A & $\mathrm{R}$ & $B, M, W$ \\
\hline 275. Song Thrush & Turdus philomelos & drozd cikelj & A & $\mathrm{R}$ & $B, M, W$ \\
\hline 276. Redwing & Turdus iliacus & mali drozd & A & $\mathrm{R}$ & $M, W$ \\
\hline 277. Fieldfare & Turdus pilaris & drozd bravenjak & A & $\mathrm{R}$ & $B, M, W$ \\
\hline 278. Dusky Thrush & Turdus eunomus & šareni drozd & $\mathrm{B}$ & & \\
\hline 279. Common Blackbird & Turdus merula & kos & A & $\mathrm{R}$ & $B, M, W$ \\
\hline
\end{tabular}




\begin{tabular}{|c|c|c|c|c|c|}
\hline English name & Scientific name & Croatian name & $\begin{array}{l}\text { Category } \\
\text { (AERC) }\end{array}$ & Occurrence & Season \\
\hline 280. Ring Ouzel & Turdus torquatus & planinski kos & A & $\mathrm{R}$ & $B, M, W$ \\
\hline $\begin{array}{l}\text { 281. Common Rock- } \\
\text { thrush }\end{array}$ & Monticola saxatilis & kamenjar & A & $\mathrm{R}$ & $\mathrm{B}, \mathrm{M}$ \\
\hline 282. Blue Rock-thrush & Monticola solitarius & modrokos & A & $\mathrm{R}$ & B \\
\hline 283. Thrush Nightingale & Luscinia luscinia & mrki slavuj & A & $\mathrm{R}$ & M \\
\hline 284. Common Nightingale & Luscinia megarhynchos & slavuj & A & $\mathrm{R}$ & $B, M$ \\
\hline 285. Bluethroat & Luscinia svecica & modrovoljka & A & $\mathrm{R}$ & $B, M$ \\
\hline 286. European Robin & Erithacus rubecula & crvendać & A & $\mathrm{R}$ & B, M, W \\
\hline 287. Rufous Scrub-robin & Erythropygia galactotes & živičnjak & A & Rare & \\
\hline 288. Black Redstart & Phoenicurus ochruros & mrka crvenrepka & A & $\mathrm{R}$ & $B, M, W$ \\
\hline 289. Common Redstart & $\begin{array}{l}\text { Phoenicurus } \\
\text { phoenicurus }\end{array}$ & šumska crvenrepka & A & $\mathrm{R}$ & $B, M$ \\
\hline 290. Whinchat & Saxicola rubetra & smeđoglavi batić & A & $\mathrm{R}$ & $B, M$ \\
\hline 291. Common Stonechat & Saxicola torquatus & crnoglava batić & A & $\mathrm{R}$ & $B, M, W$ \\
\hline $\begin{array}{l}\text { Siberian Common } \\
\text { Stonechat }\end{array}$ & S. t. maurus & sibirski batić & A & Rare & \\
\hline 292. Northern Wheatear & Oenanthe oenanthe & sivkasta bjeloguza & A & $\mathrm{R}$ & $B, M$ \\
\hline 293. Pied Wheatear & Oenanthe pleschanka & dvobojna bjeloguza & A & Rare & \\
\hline $\begin{array}{l}\text { 294. Black-eared } \\
\text { Wheatear }\end{array}$ & Oenanthe hispanica & primorska bjeloguza & A & $\mathrm{R}$ & B \\
\hline 295. Isabelline Wheatear & Oenanthe isabellina & azijska bjeloguza & A & Rare & \\
\hline 296. Spotted Flycatcher & Muscicapa striata & muharica & A & $\mathrm{R}$ & $B, M$ \\
\hline $\begin{array}{l}\text { 297. European Pied } \\
\text { Flycatcher }\end{array}$ & Ficedula hypoleuca & crnoglava muharica & A & $\mathrm{R}$ & M \\
\hline 298. Collared Flycatcher & Ficedula albicollis & bjelovrata muharica & A & $\mathrm{R}$ & $\mathrm{B}, \mathrm{M}$ \\
\hline $\begin{array}{l}\text { 299. Semi-collared } \\
\text { Flycatcher }\end{array}$ & Ficedula semitorquata & sirijska muharica & B & & \\
\hline $\begin{array}{l}\text { 300. Red-breasted } \\
\text { Flycatcher }\end{array}$ & Ficedula parva & mala muharica & A & $\mathrm{R}$ & $B, M$ \\
\hline 301. Common Firecrest & Regulus ignicapilla & vatroglavi kraljić & A & $\mathrm{R}$ & $B, M, W$ \\
\hline 302. Goldcrest & Regulus regulus & zlatoglavi kraljić & A & $\mathrm{R}$ & B, M, W \\
\hline 303. Zitting Cisticola & Cisticola juncidis & šivalica & A & $\mathrm{R}$ & B \\
\hline 304. Cetti's Warbler & Cettia cetti & svilorepa & A & $\mathrm{R}$ & $\mathrm{B}$ \\
\hline $\begin{array}{l}\text { 305. Western } \\
\text { Grasshopper-warbler }\end{array}$ & Locustella naevia & pjegavi cvrčić & A & $\mathrm{R}$ & $B, M$ \\
\hline 306. River Warbler & Locustella fluviatilis & cvrčić potočar & A & $\mathrm{R}$ & $\mathrm{B}, \mathrm{M}$ \\
\hline 307. Savi's Warbler & Locustella luscinioides & veliki cvrčić & A & $\mathrm{R}$ & $\mathrm{B}, \mathrm{M}$ \\
\hline 308. Moustached Warbler & $\begin{array}{l}\text { Acrocephalus } \\
\text { melanopogon }\end{array}$ & crnoprugasti trstenjak & A & $\mathrm{R}$ & B, M, W \\
\hline 309. Aquatic Warbler & $\begin{array}{l}\text { Acrocephalus } \\
\text { paludicola }\end{array}$ & trstenjak ševar & A & Rare & $M^{*}$ \\
\hline 310. Sedge Warbler & $\begin{array}{l}\text { Acrocephalus } \\
\text { schoenobaenus }\end{array}$ & trstenjak rogožar & A & $\mathrm{R}$ & $B, M$ \\
\hline 311. Paddyfield Warbler & Acrocephalus agricola & poljski trstenjak & A & Rare & \\
\hline
\end{tabular}




\begin{tabular}{|c|c|c|c|c|c|}
\hline English name & Scientific name & Croatian name & $\begin{array}{c}\text { Category } \\
\text { (AERC) }\end{array}$ & Occurrence & Season \\
\hline $\begin{array}{l}\text { 312. Eurasian Reed- } \\
\text { warbler }\end{array}$ & $\begin{array}{l}\text { Acrocephalus } \\
\text { scirpaceus }\end{array}$ & trstenjak cvrkutić & A & $\mathrm{R}$ & $B, M$ \\
\hline 313. Marsh Warbler & Acrocephalus palustris & trstenjak mlakar & A & $\mathrm{R}$ & $B, M$ \\
\hline 314. Great Reed-warbler & $\begin{array}{l}\text { Acrocephalus } \\
\text { arundinaceus }\end{array}$ & veliki trstenjak & A & $\mathrm{R}$ & $B, M$ \\
\hline 315. Booted Warbler & Hippolais caligata & voljić žbunjar & A & Rare & \\
\hline 316. Olivaceous Warbler & Hippolais pallida & sivi voljić & A & $\mathrm{R}$ & B \\
\hline 317. Olive-tree Warbler & Hippolais olivetorum & voljić maslinar & A & $\mathrm{R}$ & B \\
\hline 318. Melodious Warbler & Hippolais polyglotta & kratkokrili voljić & A & $\mathrm{R}$ & B \\
\hline 319. Icterine Warbler & Hippolais icterina & žuti voljić & A & $\mathrm{R}$ & $\mathrm{B}, \mathrm{M}$ \\
\hline 320. Willow Warbler & Phylloscopus trochilus & brezov zviždak & A & $\mathrm{R}$ & $B, M$ \\
\hline 321. Common Chiffchaff & Phylloscopus collybita & zviždak & A & $\mathrm{R}$ & B, M, W \\
\hline $\begin{array}{l}\text { 322. Eastern Bonelli's } \\
\text { Warbler }\end{array}$ & Phylloscopus orientalis & gorski zviždak & A & $\mathrm{R}$ & B \\
\hline 323. Wood Warbler & Phylloscopus sibilatrix & šumski zviždak & A & $\mathrm{R}$ & $B, M$ \\
\hline 324. Pallas's Leaf-warbler & $\begin{array}{l}\text { Phylloscopus } \\
\text { proregulus }\end{array}$ & zlatoglavi zviždak & A & Rare & \\
\hline $\begin{array}{l}\text { 325. Yellow-browed } \\
\text { Warbler }\end{array}$ & Phylloscopus inornatus & azijski zviždak & A & Rare & \\
\hline 326. Hume's Leaf-warbler & Phylloscopus humei & srebrnastoprsi zviždak & A & Rare & \\
\hline 327. Blackcap & Sylvia atricapilla & crnokapa grmuša & A & $\mathrm{R}$ & $B, M, W$ \\
\hline 328. Garden Warbler & Sylvia borin & siva grmuša & A & $\mathrm{R}$ & $B, M$ \\
\hline 329. Barred Warbler & Sylvia nisoria & pjegava grmuša & A & $\mathrm{R}$ & $B, M$ \\
\hline 330. Lesser Whitethroat & Sylvia curruca & grmuša čevrljinka & A & $\mathrm{R}$ & $B, M$ \\
\hline 331. Orphean Warbler & Sylvia hortensis & velika grmuša & A & $\mathrm{R}$ & $\mathrm{B}$ \\
\hline 332. Greater Whitethroat & Sylvia communis & grmuša pjenica & A & $\mathrm{R}$ & $\mathrm{B}, \mathrm{M}$ \\
\hline 333. Dartford Warbler & Sylvia undata & crvenooka grmuša & B & & \\
\hline 334. Subalpine Warbler & Sylvia cantillans & bjelobrka grmuša & A & $\mathrm{R}$ & B \\
\hline 335. Sardinian Warbler & Sylvia melanocephala & crnoglava grmuša & A & $\mathrm{R}$ & $\mathrm{B}$ \\
\hline 336. Rüppell's Warbler & Sylvia rueppelli & crnogrla grmuša & A & Rare & \\
\hline 337. Bearded Parrotbill & Panurus biarmicus & brkata sjenica & A & $\mathrm{R}$ & B, M, W \\
\hline 338. Sombre Tit & Poecile lugubris & mrka sjenica & A & $\mathrm{R}$ & B \\
\hline 339. Marsh Tit & Poecile palustris & crnoglava sjenica & A & $\mathrm{R}$ & B \\
\hline 340. Willow Tit & Poecile montanus & planinska sjenica & A & $\mathrm{R}$ & $\mathrm{B}$ \\
\hline 341. Coal Tit & Periparus ater & jelova sjenica & A & $\mathrm{R}$ & $B, M^{*}, W^{*}$ \\
\hline 342. European Crested Tit & Lophophanes cristatus & kukmasta sjenica & A & $\mathrm{R}$ & B \\
\hline 343. Great Tit & Parus major & velika sjenica & A & $\mathrm{R}$ & $\mathrm{B}, \mathrm{M}^{*}, \mathrm{~W}^{*}$ \\
\hline 344. Common Blue Tit & Cyanistes caeruleus & plavetna sjenica & $\mathrm{A}$ & $\mathrm{R}$ & $\mathrm{B}, \mathrm{W}^{*}$ \\
\hline 345. Azure Tit & Cyanistes cyanus & bijela sjenica & B & & \\
\hline $\begin{array}{ll}\text { 346. } & \text { Eurasian } \\
\text { Pendulinetit }\end{array}$ & Remiz pendulinus & sjenica mošnjarka & A & $\mathrm{R}$ & $\mathrm{B}, \mathrm{M}, \mathrm{W}$ \\
\hline
\end{tabular}




\begin{tabular}{|c|c|c|c|c|c|}
\hline English name & Scientific name & Croatian name & $\begin{array}{l}\text { Category } \\
\text { (AERC) }\end{array}$ & Occurrence & Season \\
\hline 347. Long-tailed Tit & Aegithalos caudatus & dugorepa sjenica & A & $\mathrm{R}$ & $\mathrm{B}$ \\
\hline 348. Eurasian Nuthatch & Sitta europaea & brgljez & A & $\mathrm{R}$ & B \\
\hline $\begin{array}{l}\text { 349. Western Rock } \\
\text { Nuthatch }\end{array}$ & Sitta neumayer & brgljez kamenjar & A & $\mathrm{R}$ & B \\
\hline 350. Wallcreeper & Tichodroma muraria & zidarčac & A & $\mathrm{R}$ & W \\
\hline 351. Eurasian Treecreeper & Certhia familiaris & kratkokljuni puzavac & $A$ & $\mathrm{R}$ & B \\
\hline $\begin{array}{ll}\text { 352. } & \text { Short-toed } \\
\text { Treecreeper }\end{array}$ & Certhia brachydactyla & dugokljuni puzavac & A & $\mathrm{R}$ & B \\
\hline $\begin{array}{l}\text { 353. Eurasian Golden } \\
\text { Oriole } \\
\end{array}$ & Oriolus oriolus & vuga & A & $\mathrm{R}$ & B, M \\
\hline 354. Red-backed Shrike & Lanius collurio & rusi svračak & A & $\mathrm{R}$ & $B, M$ \\
\hline 355. Lesser Grey Shrike & Lanius minor & sivi svračak & A & $\mathrm{R}$ & $B, M$ \\
\hline 356. Great Grey Shrike & Lanius excubitor & veliki svračak & A & $\mathrm{R}$ & W \\
\hline 357. Woodchat Shrike & Lanius senator & riđoglavi svračak & A & $\mathrm{R}$ & B \\
\hline 358. Eurasian Jay & Garrulus glandarius & šojka & A & $\mathrm{R}$ & $B, W$ \\
\hline 359. Common Magpie & Pica pica & svraka & A & $\mathrm{R}$ & B \\
\hline 360. Spotted Nutcracker & Nucifraga caryocatactes & kreja & A & $\mathrm{R}$ & B \\
\hline $\begin{array}{l}\text { 361. Yellow-billed } \\
\text { Chough }\end{array}$ & Pyrrhocorax graculus & žutokljuna galica & A & $\mathrm{R}$ & B \\
\hline 362. Eurasian Jackdaw & Corvus monedula & čavka & A & $\mathrm{R}$ & B \\
\hline 363. Rook & Corvus frugilegus & gačac & A & $\mathrm{R}$ & $\mathrm{B}, \mathrm{W}^{*}$ \\
\hline 364. Carrion Crow & Corvus corone & crna vrana & A & Rare & \\
\hline 365. Hooded Crow & Corvus cornix & siva vrana & $A$ & $\mathrm{R}$ & B \\
\hline 366. Common Raven & Corvus corax & gavran & A & $\mathrm{R}$ & B \\
\hline 367. Common Starling & Sturnus vulgaris & čvorak & A & $\mathrm{R}$ & $B, M, W$ \\
\hline $\begin{array}{l}\text { 368. Rose-coloured } \\
\text { Starling } \\
\end{array}$ & Pastor roseus & ružičasti čvorak & A & Ir & \\
\hline 369. House Sparrow & Passer domesticus & vrabac & A & $\mathrm{R}$ & B \\
\hline 370. Spanish Sparrow & Passer hispaniolensis & španjolski vrabac & A & $\mathrm{R}$ & B \\
\hline $\begin{array}{l}\text { 371. Eurasian Tree } \\
\text { Sparrow }\end{array}$ & Passer montanus & poljski vrabac & A & $\mathrm{R}$ & $B, M^{*}, W^{*}$ \\
\hline $\begin{array}{l}\text { 372. Common Rock- } \\
\text { sparrow }\end{array}$ & Petronia petronia & vrabac kamenjar & B & & \\
\hline $\begin{array}{l}\text { 373. White-winged } \\
\text { Snowfinch }\end{array}$ & Montifringilla nivalis & snježni vrabac & A & Rare & \\
\hline 374. Common Chaffinch & Fringilla coelebs & zeba & A & $\mathrm{R}$ & $B, M, W$ \\
\hline 375. Brambling & Fringilla montifringilla & sjeverna zeba & A & $\mathrm{R}$ & $M, W$ \\
\hline 376. European Serin & Serinus serinus & žutarica & A & $\mathrm{R}$ & $B, M, W$ \\
\hline 377. European Greenfinch & Carduelis chloris & zelendur & A & $\mathrm{R}$ & $\mathrm{B}, \mathrm{M}, \mathrm{W}$ \\
\hline 378. Eurasian Siskin & Carduelis spinus & čižak & A & $\mathrm{R}$ & $B, M, W$ \\
\hline 379. European Goldfinch & Carduelis carduelis & češljugar & A & $\mathrm{R}$ & $B, M, W$ \\
\hline 380. Citril Finch & Carduelis citrinella & planinska žutarica & A & Rare & \\
\hline
\end{tabular}




\begin{tabular}{|c|c|c|c|c|c|}
\hline English name & Scientific name & Croatian name & $\begin{array}{l}\text { Category } \\
\text { (AERC) }\end{array}$ & Occurrence & Season \\
\hline 381. Lesser Redpoll & Carduelis cabaret & alpska juričica & A & $\mathrm{R}$ & W \\
\hline 382. Common Redpoll & Carduelis flammea & sjeverna juričica & A & $\mathrm{R}$ & W \\
\hline 383. Twite & Carduelis flavirostris & gorska juričica & A & Rare & \\
\hline 384. Common Linnet & Carduelis cannabina & juričica & A & $\mathrm{R}$ & $B, M, W$ \\
\hline 385. Common Rosefinch & Carpodacus erythrinus & rujnica & A & Rare & \\
\hline 386. Red Crossbill & Loxia curvirostra & krstokljun & A & $\mathrm{R}$ & $\mathrm{B}, \mathrm{M}^{*}, \mathrm{~W}^{*}$ \\
\hline 387. Parrot Crossbill & Loxia pytyopsittacus & veliki krstokljun & B & & \\
\hline 388. Eurasian Bullfinch & Pyrrhula pyrrhula & zimovka & A & $\mathrm{R}$ & $B, M^{*}, W$ \\
\hline 389. Hawfinch & $\begin{array}{l}\text { Coccothraustes } \\
\text { coccothraustes }\end{array}$ & batokljun & A & $\mathrm{R}$ & $B, M, W$ \\
\hline 390. Lapland Longspur & Calcarius lapponicus & laponska strnadica & A & Rare & \\
\hline 391. Snow Bunting & Plectrophenax nivalis & bijela strnadica & A & Rare & \\
\hline 392. Corn Bunting & Emberiza calandra & velika strnadica & A & $\mathrm{R}$ & $B, M, W^{*}$ \\
\hline 393. Yellowhammer & Emberiza citrinella & žuta strnadica & A & $\mathrm{R}$ & $\mathrm{B}, \mathrm{M}^{*}, \mathrm{~W}^{*}$ \\
\hline 394. Pine Bunting & $\begin{array}{l}\text { Emberiza } \\
\text { leucocephalos }\end{array}$ & bjeloglava strnadica & A & Rare & \\
\hline 395. Rock Bunting & Emberiza cia & strnadica cikavica & A & $\mathrm{R}$ & B \\
\hline 396. Ortolan Bunting & Emberiza hortulana & vrtna strnadica & A & $\mathrm{R}$ & $B, M$ \\
\hline 397. Cirl Bunting & Emberiza cirlus & crnogrla strnadica & $\mathrm{A}$ & R & B \\
\hline 398. Little Bunting & Emberiza pusilla & mala strnadica & A & Rare & \\
\hline 399. Rustic Bunting & Emberiza rustica & šumska strnadica & A & Rare & \\
\hline $\begin{array}{l}\text { 400. Black-headed } \\
\text { Bunting }\end{array}$ & $\begin{array}{l}\text { Emberiza } \\
\text { melanocephala }\end{array}$ & crnoglava strnadica & A & $\mathrm{R}$ & B \\
\hline 401. Reed Bunting & Emberiza schoeniclus & močvarna strnadica & A & $\mathrm{R}$ & $B, M, W$ \\
\hline
\end{tabular}

Escapes and probable escapes (Category E, D)

\begin{tabular}{|l|l|l|c|}
\hline - Barbary Partridge & Alectoris barbara & afrička jarebica & E \\
\hline - Red-legged Partridge & Alectoris rufa & riđa jarebica & E \\
\hline - Muscovy Duck & Cairina moschata & mošusna patka & E* \\
\hline - Wood Duck & Aix sponsa & američka mandarinka & E \\
\hline - White-cheeked Pintail & Anas bahamensis & smeđokapa patka & E \\
\hline - Chilean Flamingo & Phoenicopterus chilensis & čilski plamenac & E \\
\hline - Yellow-billed Stork & Mycteria leucocephala & šarena roda & E \\
\hline - African Spoonbill & Platalea alba & afrička žličarka & D \\
\hline - Northern Bald Ibis & Geronticus eremita & ćlavi ibis & E \\
\hline - Lappet-faced Vulture & Torgos tracheliotos & veliki sup & E \\
\hline - Rose-ringed Parakeet & Psittacula krameri & mala aleksandra & E \\
\hline - Yellow-crowned Bishop & Euplectes afer & žutokruna pletilja & E \\
\hline
\end{tabular}




\begin{tabular}{|l|c|c|c|c|}
\hline Summary & $\begin{array}{c}\text { Category } \\
\text { (AERC) }\end{array}$ & Season & Occurrence & \\
\hline Species total & A, B, C & all & all & 396 \\
\hline Recorded in a wild state since 1.1.1800 & A, B & all & all & 393 \\
\hline Recorded in a wild state since 1.1.1950 & A & all & all & 374 \\
\hline self-supported populations derived from introduced stock & C & all & all & 3 \\
\hline Regular breeding species & A, C & B & R & 230 \\
\hline - autochthonous breeders & A & B & R & 227 \\
\hline - introduced breeders & C & B & R & 3 \\
\hline Irregular breeding species & A & B* & Ir, R & 5 \\
\hline Regular non-breeding species & A & M, W & R & 55 \\
\hline Irregular visitors & A & all & Ir & 21 \\
\hline Genuine vagrants & A & & Rare & 67 \\
\hline $\begin{array}{l}\text { Coming from self-supported populations derived } \\
\text { from introduced stock or escapees }\end{array}$ & C E & & & 5 \\
\hline $\begin{array}{l}\text { Probable escapees but genuine vagrancy could } \\
\text { not be excluded }\end{array}$ & D & & & 1 \\
\hline Escapees & E & & & 11 \\
\hline
\end{tabular}




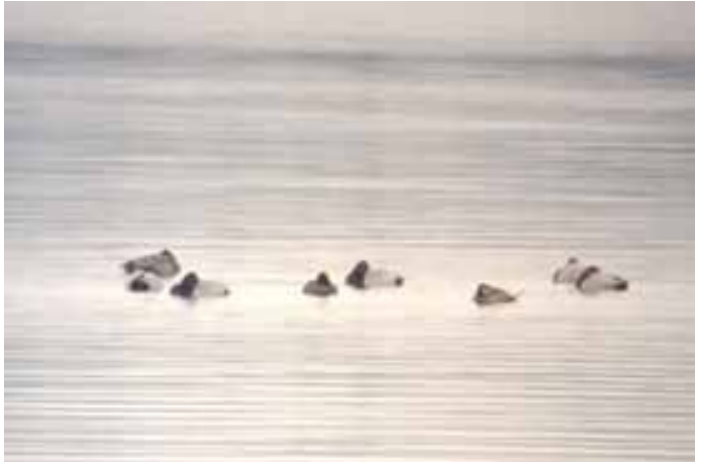

Figure 1. White-headed Duck Oxyura leucocephala (photo: D. Bordjan)

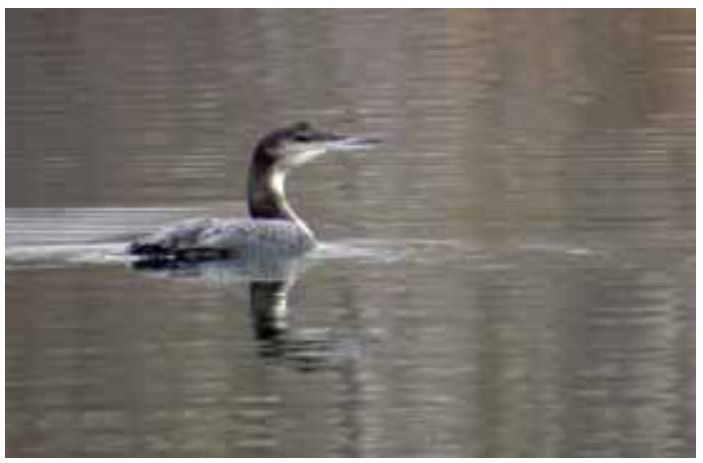

Figure 3. Common Loon -

Gavia immer (photo: L. Hercigonja)

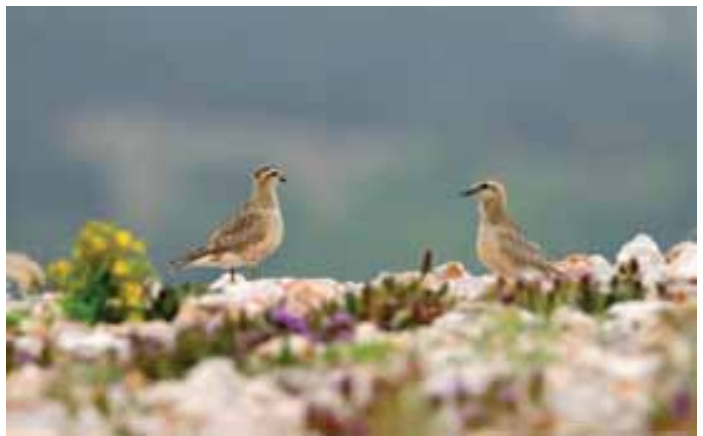

Figure 5. Eurasian Dotterel -

Eudromias morinellus (photo: T. Klanfar)

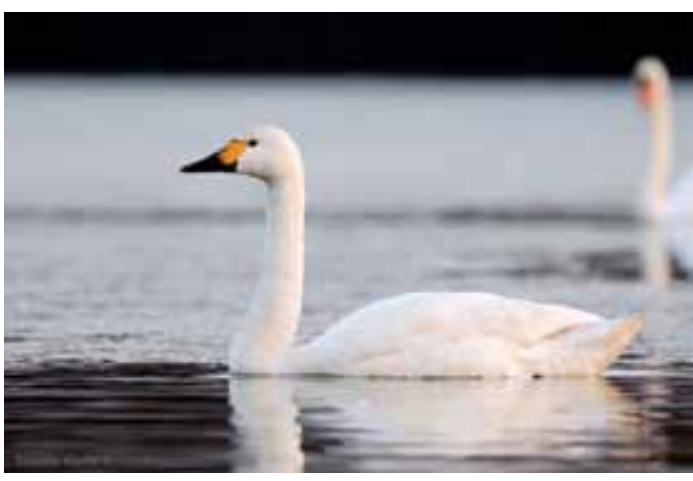

Figure 2. Tundra Swan Cygnus columbianus (photo: T. Klanfar)

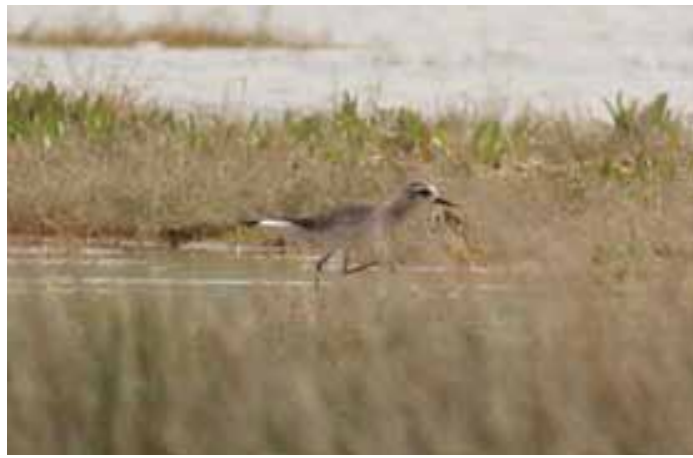

Figure 4. American Golden Plover Pluvialis dominica (photo: A. Ploj)

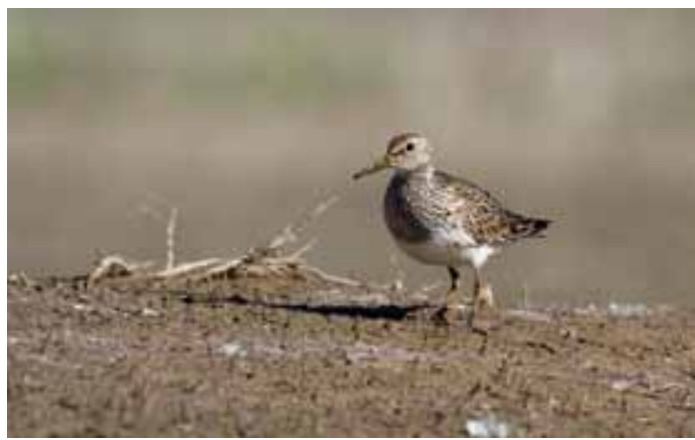

Figure 6. Pectoral SandpiperCalidris melanotos (photo: I. Lolić) 


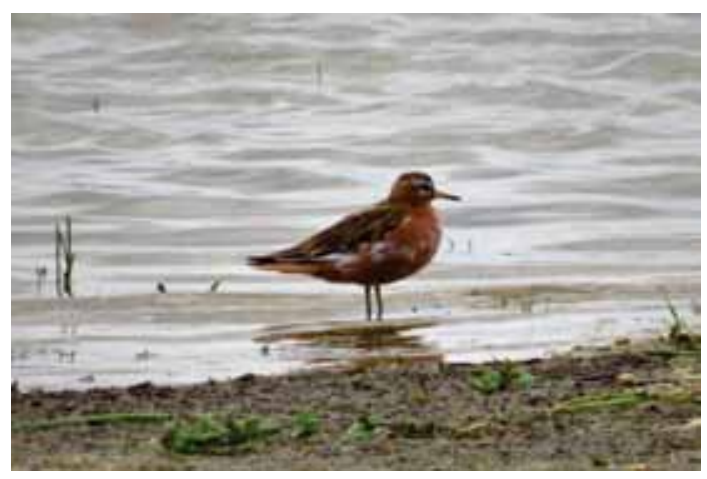

Figure 7. Red Phalarope -

Phalaropus fulicarius (photo: Z. Tkalčec)

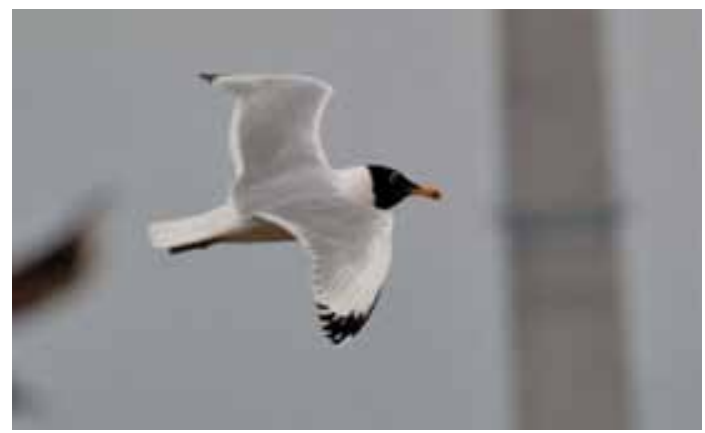

Figure 9. Pallas's Gull -

Larus ichthyaetus (photo: L. Jurinović)

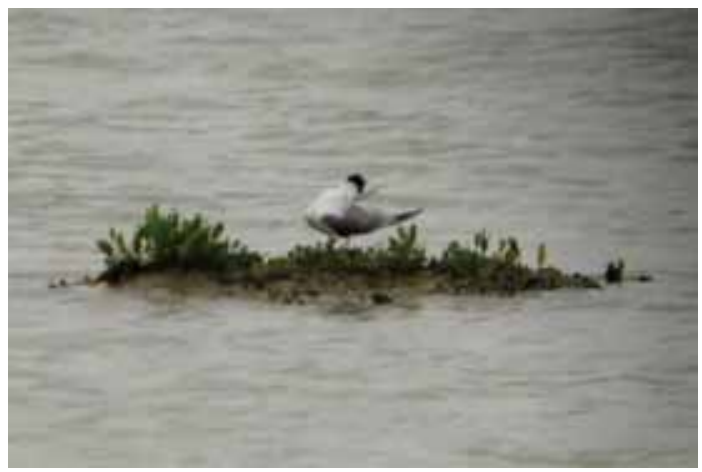

Figure 11. Lesser Crested Tern -

Thalasseus bengalensis (photo: P. Tout)

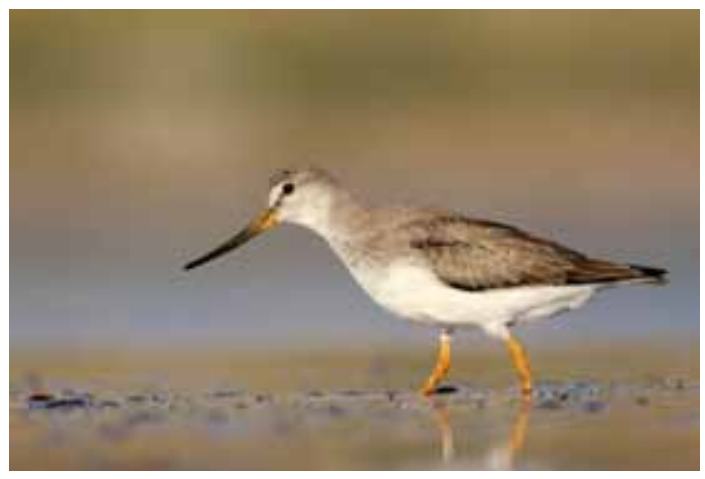

Figure 8. Terek Sandpiper -

Xenus cinereus (photo: A. Ploj)

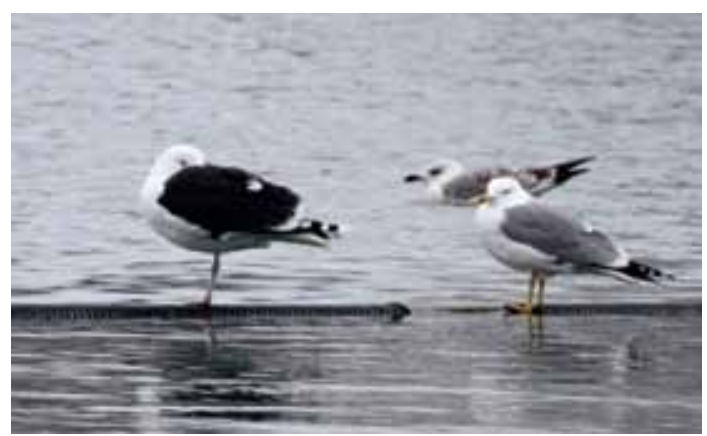

Figure 10. Great Black-backed Gull Larus marinus (photo: M. Szymański)

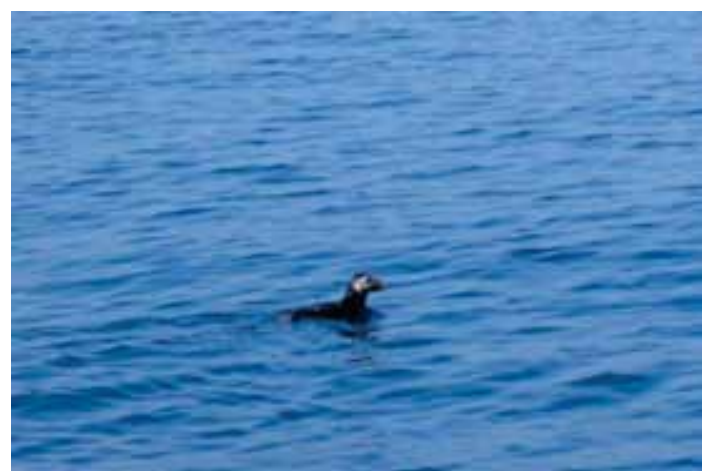

Figure 12. Atlantic Puffin -

Fratercula arctica (photo: N. Alač) 

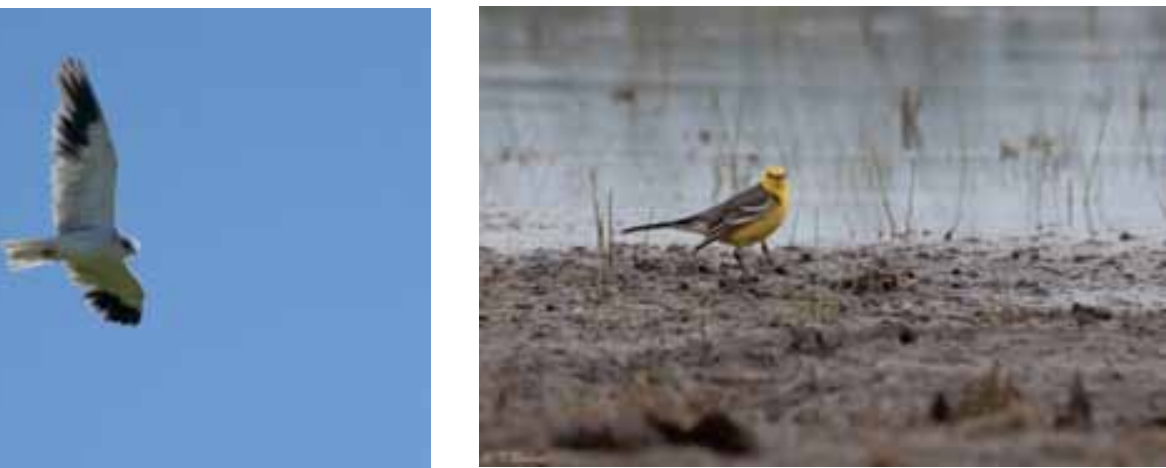

Figure 13. Black-winged Kite Elanus caeruleus (photo: I. Dević)

Figure 14. Citrine Wagtail -

Motacilla citreola (photo: T. Rubinić)

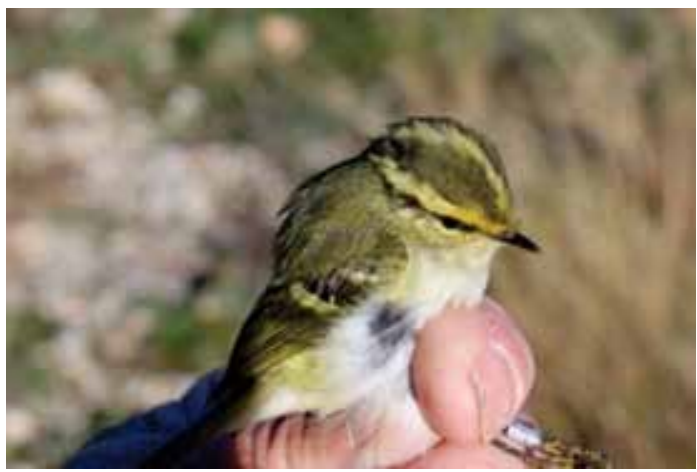

Figure 15. Paddyfield Warbler -

Acrocephalus agricola (photo: I. Lolić)

Figure 16. Pallas's Leaf-warbler -

Phylloscopus proregulus (photo: P. Corva)

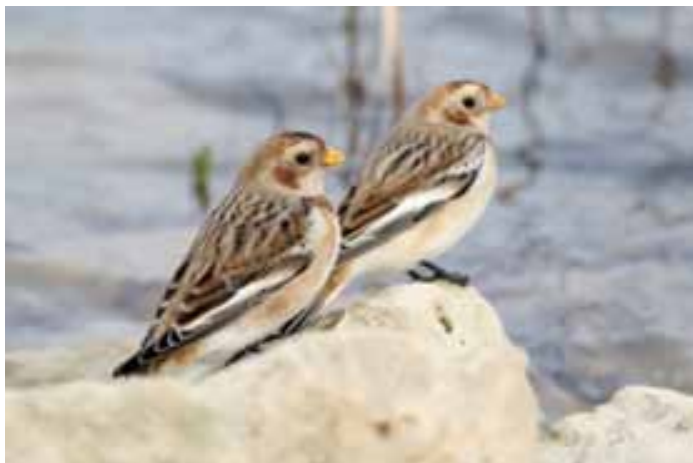

Figure 18. Snow Bunting -

Plectrophenax nivalis (photo: M. Szymański) 